\title{
A Review of Post-Prognostics Decision-Making in Prognostics and Health Management
}

\author{
Omar Bougacha ${ }^{1}$, Christophe Varnier ${ }^{2}$ and Noureddine Zerhouni ${ }^{3}$ \\ 1,2,3 FEMTO-ST, AS2M, \\ Univ. Bourgogne Franche-Comté, ENSMM, \\ Besançon, 25000, France \\ omar.bougacha@femto-st.fr \\ christophe.varnier@femto-st.fr \\ noureddine.zerhouni@femto-st.fr
}

\begin{abstract}
Mainly, the prognostics and health management (PHM) process is based on three processes: the data acquisition and health assessment process in which sensors signals are acquired and processed, the diagnostic and prognostic process in which the source of failure is detected and the remaining useful life (RUL) is predicted and finally the decisionmaking process that refers to the term management in prognostics and health management. This paper reviews in the literature about the different aspects of decision-making in the context of PHM. The selected papers are subject to content assessment and grouped according to the decision type. Additionally, this paper presents a synthesis of the previous works that helps identify new trends and deficiencies in the decision-making process. The synthesis can guide efforts for future work.
\end{abstract}

\section{INTRODUCTION}

Industries exist in highly competitive environments. To maintain their profit margins, they need a high level of system reliability and availability at the lowest cost. To control the systems' dependability, an adequate maintenance policy needs to be set up. Thus, maintenance policies have evolved quickly from waiting to fix the machine after it fails in a corrective maintenance style to preventive maintenance. In its turn, preventive maintenance has evolved from systemic maintenance, in which the interventions were scheduled using age-dependent metrics or reliability information provided by the component constructor, to condition-based maintenance (CBM). As the name suggests, in CBM, we assess the condition of a component, i. e. , its degradation level, and we follow its evolution until it crosses a threshold, at which point

\footnotetext{
Omar Bougacha et al. This is an open-access article distributed under the terms of the Creative Commons Attribution 3.0 United States License, which permits unrestricted use, distribution, and reproduction in any medium, provided the original author and source are credited.
}

a maintenance action is scheduled. Lately, with the introduction of prognostics (Vachtsevanos \& Wang, 2001), defined as the science of prediction in (Goebel et al., 2017), CBM emerged into prognostics and health management (PHM) as described in (Byington, Roemer, \& Galie, 2002). As a result, one no longer compares the actual degradation level to a threshold. Instead, we estimate the remaining useful life (RUL) of the component in question by predicting its end-of-life (EoL). Based on RUL, a decision should be made to avoid failures.

One can finds plenty of definition for the PHM concept in literature, but no unified definition has yet been proposed. In (Uckun, Goebel, \& Lucas, 2008), Uckrun et et al. defined PHM as an engineering discipline that joins the study of the failure mechanism and the management of the system's life cycle. In (Sun, Zeng, Kang, \& Pecht, 2010), Sun et al. described PHM as a methodology to not only predict the component responsible for the failure and when it will fail, but to also reduce the risks by studying the reliability of a system in its environmental, operational and usage conditions. The Center for Advanced Life Cycle Engineering (CALCE 2012) defined PHM as "the means to predict and protect the integrity of equipment and complex systems and avoid unanticipated operational problems leading to mission performance deficiencies, degradation and adverse effects to mission safety. " Skima in (Skima, 2016), used the same definition as Sun et al. but specified that the objective behind the use of PHM systems is to manage the health of a system by minimizing its operations and maintenance costs. Goebel et al. in (Goebel et al., 2017), did not directly define PHM, instead defining prognostics in the PHM context as when one is studying the conditions of a fielded engineering system and whether it is behaving within nominal operation bounds. If it is not, one then predicts where and when the system will no longer fulfill certain functional requirements. They specify that the prognostics are performed not as an objective in 
themselves, but for a decision-making process afterward.

The PHM process is similar to the architecture presented in the OSA/CBM in (Lebold \& Thruston, 2001). Open standard implies that a PHM or CBM system should be built over seven layers: data acquisition, data processing, condition assessment, diagnostics, prognostics, decision-making and human machine interface. Lately, these layers have been grouped into three processes in (Gouriveau, Medjaher, \& Zerhouni, 2016): the observation process that contains data acquisition and processing; the analysis process in which the system's conditions will be assessed, followed by diagnostics and prognostics; and finally, the act process involve decisionmaking and application through the human-machine interface.

According to the origin of PHM, one can define a decision as a set of maintenance actions. This decision is usually the solution to an optimization problem consisting in defining adequate interventions dates. But the use of prognostics has evolved to include other sorts of decisions. For example, prognostics information were used to define process set points and controller parameters in closed-loop actuators controls as mentioned in (Pereira, Galvão, \& Yoneyama, 2010) or (Langeron, Grall, \& Barros, 2013). Other works have integrated prognostics information into production scheduling and mission assignment, in which the health prognostic of the system was defined as a constraint or included in the optimization objective, as was the case in the works of (Herr, Nicod, Varnier, Jardin, et al., 2017).

Gouriveau et al. $\quad$ stated in (Gouriveau et al., 2016) that the PHM is not limited to industrial maintenance but can be applied to any kind of activity as long as it fits the elementary process defined by the OSA/CBM decomposition. The only difference is in the nature of the decisions to be made. The evolving nature of the integration of prognostics information in different kinds of decisions calls for a review of the existing works in this domain. Consequently, this paper investigates the approaches to decision-making for all kinds of decisions, the proposed scope of work, the dominant assumptions and the fields of application. Next, current and future challenging issues are pointed out and discussed to help centralize efforts for future works. Although it is important to note that this paper does not present an exhaustive review because we limited our study to papers published between 2006 and 2018.

The organization of the paper is as follows: a definition of post-prognostics decisions is presented in Section 2, with a presentation of the bibliographic search results. In Sections 3,4 and 5, related works are classified by the type of decisions and discussion is, also, presented for each type of decisions to compare the approaches proposed in those papers. Section 6 presents a synthesis of the selected papers, new trends and scientific locks. Finally, we present a conclusion of this study and suggest some future works in Section 7.

\section{Definition of Post-Prognostic Decisions}

Literally decision-making is the name given to the process of selecting the logical best choice from a list of available options. In such a process one should weight the pros and cons of each possible option, consider all the alternatives and forecast the outcome of each option. Such a process can either be performed by human operators or a systemic approach that can support the human operators decision-making process. As a result, one can model the decision-making problem with an optimization problem. Since human decision-makers are limited in their cognitive capacity to process and analyze large quantities of information, in the context of industry, the decision-making process will be performed by an algorithm.

In the PHM context, we are supposed to take the adequate actions to prevent or mitigate the degradation of the studied system. Thus the ultimate goal of PHM is to make decisions based on prognostics information. Iyer et al. in (Iyer, Goebel, \& Bonissone, 2006) were the first to introduce the term "post-prognostic decisions" to describe decisions made in the PHM context using prognostic information from the system to choose the most adequate actions for logistics platforms, maintenance, supply chain management, mission planning and mission allocation. Later, Balaban et al. in (Balaban \& Alonso, 2012), defined the post-prognostic decision as one that takes into consideration the prognostic information (i. e. , RUL) to define the future utilization of the system in question so as to optimize a predefined objective. This same definition was used by Herr in (Herr, 2015) and by Chebel-Morello et al. in (Chebel-Morello, Nicod, \& Varnier, 2017). Recently in (Goebel et al., 2017), Goebel et al. gave a more precise definition for post-prognostic decisions. As a result, one calls a post-prognostic decision the set of actions at time $t$ that best overcome an undesirable future event, predicted to take place at $t+t_{E}$, by satisfying a given set of constraints and optimizing a set of objectives expressed in a cost function.

In the same context, we propose that post-prognostic decisions are a sequence of configurable actions built over a predefined duration, called the decision horizon. This series of actions is built at time $t$ to best manage the health of the system, its operations and its missions under the prospect of an undesirable future event, foreseen to take place at $t+t_{E}$. The built decision must satisfy a given set of constraints and optimize a set of objectives expressed in a cost function.

In the literature, one can find plenty of works that discuss post-prognostic decision-making. To study these articles, one can conduct a two-level bibliographical research. First, different combinations of keywords related to prognostics and decision-making, presented in Table 1, can be used in classic search engines like Web-of-Science and Google Scholar. Sorting the resulting papers is the next step to identify the relevant ones. Then one can search for the works that cited the 
outstanding studied papers. Using this method, we identified a total of 109 articles (see Figure 1). The growing number of publications per year proves that post-prognostic decision is an expending research domain. Also we note the absence of papers that review the integration of prognostics information in decision-making and the importance such a paper can have in defining the current and future challenges in postprognostic decision-making.

Table 1. The list of the used keywords.

\begin{tabular}{c}
\hline Keywords \\
\hline Prognostics information \\
Post-Prognostic decision-making \\
Maintenance decisions \\
Maintenance scheduling \\
Maintenance planning \\
Operational decisions \\
Operational planning \\
Joint optimization \\
PHM decision-making \\
Condition-Based Maintenance \\
\hline
\end{tabular}

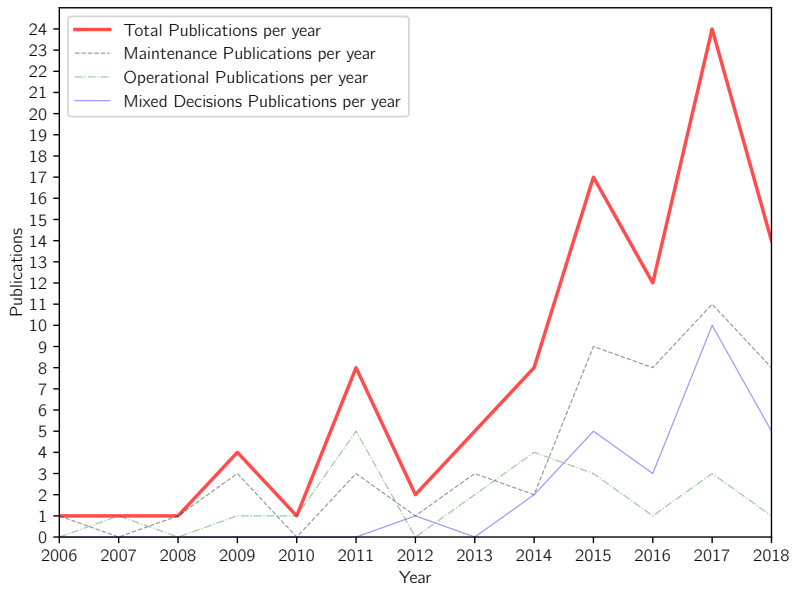

Figure 1. Evolution of publications on Post-Prognostic Decision

In (Goebel et al., 2017), Goebel et al. proposed a classification of the possible prognostic-based actions to take depending on the duration of the prognostic horizon. The classification included controller reallocation, mission rescheduling, and maintenance plan optimization. In this paper, we propose another classification of the post-prognostic decisions based on the nature of the action itself. The analysis of the type of decisions discussed in the identified papers helped define the categories. As a result, we propose to classify post-prognostic decisions into three categories using the type of the decision as a criterion: $(i)$ decisions that describes the schedule of maintenance activities, $(i i)$ decision that influence the operational condition of the system and $(i i i)$ mixed decisions that optimize jointly the maintenance of the system and its operational conditions. Table 2 represents the distribution of arti- cles according to these three categories. One can easily note that the maintenance decisions are more dominant. indeed, operational and mixed decisions are more recent fields of research. These categories are explored in the next sections, in which a further analysis is conducted on subcategories and on the related work by reviewing their ideas and investigating their main assumptions.

Table 2. Publications on Post-Prognostic Decision per Decision Type

\begin{tabular}{cccc} 
Type of Decision & Maintenance & Operational & Mixed \\
\hline Publications & 51 & 28 & 30 \\
Percentage & $46.8 \%$ & $25.5 \%$ & $27.7 \%$
\end{tabular}

\section{Maintenance Decisions}

In this section, the works that considered maintenance scheduling and maintenance interventions optimization based on prognostics information are studied. In the literature, postprognostics maintenance planning can be divided into two categories according to the number of the considered machines. Single machine maintenance planning and multiple machine maintenance scheduling are detailed in the following subsections. In each category, the authors solved the maintenance scheduling problem for either multi-machine or single machine system configuration. They assumed that a machine is composed of one or more components, and that the health status or the degradation of the system is known. A prognostic algorithm provides the predictive information i. e. the remaining useful life, the evolution of degradation, or the probability of failure. Based on the estimated future information, the authors proposed methods to optimize a given objective function under several constraints that both depends on the type of application.

\subsection{Single Machine Maintenance Planning}

In this subsection, the authors focused on single machine maintenance scheduling based on the estimated health condition of the machines' components. The papers that focused on one machine maintenance planning are grouped according to the application.

\subsubsection{Manufacturing}

Zhao et al. (Zhao, Fouladirad, Bérenguer, \& Bordes, 2009) presented a new method for optimizing the maintenance policy for a system with a single deterioration, to minimize long-run maintenance costs. Camci used genetic algorithms in (Camci, 2009) to schedule maintenance interventions for a multiple-component single machine. The schedule developed by this method minimizes the maintenance cost and satisfies the constraints on available resources. Tian et al. (Tian \& Liao, 2011) considered the maintenance scheduling 
of a multiple-component single system in which the identical components are economically dependent. In this paper, Tian et al. used the proportional hazard model instead of RUL to estimate failure probability. Based on the obtained failure probability, suitable maintenance actions are made. Van Horenbeeek and Pintelon in (Van Horenbeek \& Pintelon, 2013) scheduled the maintenance activities of a multiplecomponent single machine on a finite rolling horizon. The proposed approach consists of selecting the suitable dates for maintenance interventions and then updating them when new prognostics information is available. In addition, a grouping algorithm was used to group maintenance activities of the different components based of their dependencies. The three considered components that presented different kinds of dependencies. Finally, the paper presented the effects of dependencies of the component lifetime with different maintenance policies. In the same context of a rolling decision horizon, Rodrigues et al. (Ramos Rodrigues, Paixao de Medeiros, \& Strottmann Kern, 2015) proposed a dynamic maintenance strategy for a group of similar components monitored with a PHM system. At each inspection time, the prognostics information of the components is estimated. Based on the failure probabilities of the components a two-level decision-making process is executed. At the first level the cost of maintenance of each component is minimized. Next, the maintenance activities are grouped to minimize the overall maintenance cost. The obtained schedule is applied until the next inspection, when new health information is available and the rolling horizon is shifted. Khoury et al. (Khoury, Deloux, Grall, \& Bérenguer, 2013) presented a framework for predictive maintenance of a single machine over a finite rolling "visibility" horizon. The decision horizon is characterized by two maintenance opportunities in which maintenance actions could be exclusively planned. The main objective is to find a trade-off between doing premature maintenance at the first opportunity or planning the intervention too late at the second opportunity or later based on a failure probability obtained from the RUL distribution. If the failure probability at the maintenance opportunity exceeds a certain threshold, then a maintenance action is scheduled in that opportunity; otherwise, it is postponed. Huynh et al. in (K. T. Huynh, Castro, Barros, \& Berenguer, 2014) studied the case of a single-component single machine with aging effects. In this paper, the machine could fail due to degradation or to the effect of a shock caused by the accumulation of degradation and the aging of the machine. For this reason, Huynh et al. proposed two maintenance policies based on the rate of the machine's degradation and the mean residual life. In (K. Huynh, Grall, \& Bérenguer, 2017), Huynh et al. not only proposed unusual new prognostic-based maintenance strategies built on various variants of the systems RUL, but also compared these strategies to diagnostic-based strategies and $\mathrm{CBM}$ and then quantified the performance and robustness of the diagnostic and prognostic indicators for maintenance decision-making. Tang et al. (D. Tang, Makis, Jafari, \& Yu, 2015) proposed two control limit maintenance policies based on the current degradation of a single component machine. The first policy consists of periodic inspections using a CBM approach, while the second policy consists of delaying the first inspection time under the assumption that a slowly degrading system is less likely to cross the failure threshold at an early stage. The paper also presented a way to estimate the RUL of the machine, but this RUL was not integrated into the decision-making process. Do et al. (Do, Voisin, Levrat, \& Iung, 2015) used a gamma process to describe the degradation of the system. The authors proposed an inspection-based CBM strategy with the possibility of choosing the quality of the preventive maintenance intervention. This work also presents a novel method for the definition of the inspection dates based on the RUL of the system. The failure probability of the system is estimated from its RUL, and the next inspection is scheduled to guarantee that the failure probability does not exceeds a required reliability level. Langeron et al. (Langeron, Fouladirad, \& Grall, 2016) applied the maintenance scheduling on a controlled system subject to a random deterioration of the actuator. The maintenance optimization was carried out on the controller settings along with the RUL threshold and the inspection period, at which point two possible actions were considered: either a preventive replacement or a corrective one. Shi et al. (Shi \& Zeng, 2016) considered the case of stochastic dependencies of components when the degradation of a component affects the degradation of another. The proposed approach in this paper used a dynamic opportunistic maintenance scheduling based on a real-time RUL prediction. Liu et al. (Q. Liu, Dong, Lv, \& Ye, 2017) developed a new approach for maintenance intervention scheduling on a manufacturing machine to optimize the maintenance cost based on prognostics information. The approach consists of dividing the health state of the machine into four levels with two different maintenance actions. The problem is then solved using dynamic programming model. Wang et al. (K. Wang, Tian, Pecht, \& Xu, 2015) applied a PHM framework to electro-mechanical systems to make decision about components refurbishment policy. Actually refurbishment consists of making component replacements to restore the system to satisfy its initial specification. In other words refurbishment could be considered maintenance intervention by replacement. The method is based on the estimated RUL of the considered system and its components to define an optimal replacement policy for the deteriorating components while considering the impact of such policies on the system's health for the purpose of minimizing the refurbishment cost.

\subsubsection{Wind Turbines}

Lei et al. (Lei \& Sandborn, 2016), studied maintenance decision-making based on PHM information applied to wind 
turbines. The approach consists of using real options analysis to schedule maintenance action. To take uncertainty into account, the authors used simulation paths by which each path represents one possible future scenario to finally determine the optimum predictive maintenance opportunity. Mazidi et al. (Mazidi, Bertling Tjernberg, \& Sanz Bobi, 2017), presented a hybrid method of neural networks and proportional hazard model to wind turbine behavior for prognostics and maintenance management. The approach evaluates the efficiency of previously applied maintenance plans. First, a neural network is built to model the normal behavior of the wind turbine with data gathered from a supervisory control and data acquisition (SCADA) system. Then a deviation signal that describes the stress condition and the health status of the wind turbine is extracted by comparing the real-time data to the neural network predictions. The obtained signal is used to assess the goodness of previous maintenance actions and propose suggestions for future maintenance planning. This work actually takes into consideration the effect of decisions on the system.

\subsubsection{Aerospace}

The aerospace domain is intensively studied in the case of single machine systems. Si et al. ( $\mathrm{Si}, \mathrm{Li}, \mathrm{Zhang}, \& \mathrm{Hu}$, 2018) proposed a CBM replacement strategy for stochastically deteriorating aircraft components. The framework consists of a classical periodic inspection CBM policy, and even although the reliability of the components is computed it was not considered in the decision-making process. Instead, decisions are made on the current observed degradation level. In (Vianna \& Yoneyama, 2018), the authors used a Kalmannfilter-based prognostic method to estimate the future degradation and RUL of a multi-component redundant system while assuming different wear profiles. The obtained prognostic information from the multiple model of prognostics is used to specify the maintenance intervention dates that minimize the maintenance cost. Several operational aspects were considered in this work by integrating their cost into the maintenance cost. Wang et al. (Y. Wang et al., 2017) used a modelbased prognostic framework to monitor and estimate the evolution of the size of cracks in the fuselage of an aircraft. The PHM process is done periodically through inspections and decision-making. The decision-making process includes the obtained prognostic information to schedule the maintenance intervention in the current available stop or to postpone it to the next one if the safety level allows it. The maintenance cost is minimized in this framework by finding a trade-off between the probabilities of failure occurrence and the waste of RUL of the fuselage. Later in (Y. Wang et al., 2018), the authors integrated unscheduled maintenance to work in tandem with the scheduled opportunities and considered the engine and some non-structural components. Nguyen and Medjaher (K. T. Nguyen \& Medjaher, 2019) provided a full data driven framework to schedule maintenance from data acquisition to decision-making. The authors used long short term memory neural networks to predict the failure probabilities of the machine under the proposed schedule of operations. Then, they used a rule based heuristic to schedule the maintenance activities. Uncertainty in prediction have been discussed and their effects on the maintenance decision-making process have been given.

\subsubsection{Railways}

Lin et al. (S. Lin, Zhang, \& Feng, 2016) used a partially observable Markov decision Process (POMDP) to plan maintenance interventions for a traction power supply based on its RUL. The degradation of the power supply is modeled by Gauss-Poisson process, in which natural and sudden deterioration are considered. Health indicators are deduced from the degradation to define the health of the system. The POMDP is solved using a one-pass algorithm that is based on n-computation through dynamic programming. In (D. Feng, Lin, He, \& Sun, 2017), the authors developed a novel framework that combines PHM and active maintenance to better manage the health and maintenance activities of high speed railways' traction power supply system. The proposed framework consists of classical PHM technology in which RUL, reliability and risk are estimated from different data resources. Active maintenance is used in the PHM decision-making module. This module has the objective to select the suitable maintenance strategy for each component of the system (CBM, corrective, periodic preventive or RUL-based predictive) to avoid accidents and guarantee a predefined reliability requirement.

\subsubsection{Other type of applications}

Nzukam et al. (Nzukam, Voisin, Levrat, Sauter, \& Iung, 2017) proposed a maintenance decision support system for heating, ventilation and air-conditioning (HVAC) of non residential buildings. The method considers the components' RUL and their criticality on the one hand and the planned stoppages of the system on the other. The RUL distributions of each component are estimated and compared to the stoppage dates to produce a list of opportunities for maintenance actions. A grouping algorithm takes into account the RUL, the maintenance duration and the severity of each component and the duration of the maintenance opportunities, to produce a schedule of grouped maintenance interventions. Later on, the authors integrated uncertainties into their model in (Nzukam, Voisin, Levrat, Sauter, \& Iung, 2018) by supposing that the duration of the stoppages are stochastically defined. Effects of the uncertainties were studied and the consistency of their method was proved using Monte Carlos simulations.

Later, and still in single machine maintenance optics, a new structure of maintenance decision-making is proposed. In- 
stead of using a central unique maintenance decision-making process, these papers proposed to use a two-level method for maintenance scheduling. Huynh et al. (K. T. Huynh, Barros, \& Berenguer, 2015), proposed a two-level maintenance decision-making for multi-component complex systems. The novel RUL-based maintenance scheduling framework combines the system-level maintenance decisions and the component-level one. A periodic inspection is implemented, and at each inspection, the component's degradation level is updated and corresponding new RULs are estimated. Then the RUL of the system is determined by the $k-$ out $-o f-n: F$ structure. If the system's RUL fall below a predefined threshold, at the system level, an intervention should be made, and so the component level of the framework is triggered. At this level, a list of the components to maintain is made based on the components' RULs and the economic dependencies using opportunistic maintenance strategies. Nguyen et al. in (K.-A. Nguyen, Do, \& Grall, 2015) used the same concept of the framework presented in (K. T. Huynh et al., 2015), except they improved the framework by implementing the use of reliability obtained from the RUL distribution instead of using the RUL directly. Also in this updated framework, the objective at the component level is now defined as selecting and grouping the components to be maintained based on their predicted reliability and economic and structural dependencies while the system level remains unchanged by comparing the reliability of the system to a threshold. Unlike the previous works, Verbert et al. (Verbert, De Schutter, \& Babuška, 2017) started by optimizing the maintenance strategy at the component level and then moved to the system level. First, for each component, the maintenance options are evaluated considering the risk tolerance and the predicted degradation level, and then the optimal option is chosen. After choosing the maintenance option for each component, at the system level, the maintenance strategy is optimized to account for economic and structural dependencies and the component's chosen maintenance options.

\subsubsection{Synthesis}

Tables 6 and 7 describe these papers by specifying if they considered multi-components, the degradation model, the objective of the optimization and the method, how the RUL of the components/systems is used and the corresponding application domain. Authors that considered post-prognostic maintenance planning for single machine have mostly (18 papers out of $28(64.3 \%)$ ) studied multi-component systems. One can also see that stochastic processes, especially the gamma process, are extensively used as a degradation model for single machine maintenance decision-making compared to other models (see Table 3).

Except for two works that aimed to maximize either the reliability or the lifetime of the system, all works aimed at min-
Table 3. Statistic Summary of Degradation Models for Single Machine Maintenance Planning

\begin{tabular}{ccc} 
Type of Model & Publications & Percentage \\
\hline Stochastic Process & 12 & $42.9 \%$ \\
\hline Data Driven & 4 & $14.3 \%$ \\
\hline Physic Based & 2 & $7.1 \%$ \\
\hline Other & 4 & $14.3 \%$ \\
\hline Not Mentioned & 6 & $21.4 \%$ \\
\hline
\end{tabular}

imizing maintenance cost. In most of the works, RUL is used in the decision-making process to determine the dates of maintenance interventions or/and as a term in the cost function (see Table 4 Note that the sum is not equal to $100 \%$ because some works use more that one method of integration).

Table 4. Statistic Summary of RUL Integration for Single Machine Maintenance Planning

\begin{tabular}{ccc} 
Method of Integration & Publications & Percentage \\
\hline Maintenance Date & 13 & $40.4 \%$ \\
\hline Cost Function & 14 & $50 \%$ \\
\hline Strategy/Opportunity Selection & 6 & $21.4 \%$ \\
\hline Not Integrated & 3 & $10.7 \%$ \\
\hline
\end{tabular}

Most of these works actually do not present an optimization method other than a simple algorithm that compares the RUL to a safety threshold to schedule the maintenance intervention, or in the case of multiple components, the authors usually use a grouping algorithm to group the maintenance activities on the different component, assuming that maintaining two or more components at the same time costs less than maintaining each component alone.

Table 5 presents the distribution of the application domain of the found works. One can notice that half of the existing works on single machine maintenance scheduling are made in the context of manufacturing application.

Table 5. Statistic Summary of Application Context for Single Machine Maintenance Planning

\begin{tabular}{ccc} 
Application Domain & Publications & Percentage \\
\hline Manufacturing & 14 & $50 \%$ \\
\hline Aerospace & 5 & $17.9 \%$ \\
\hline Railways & 3 & $10.8 \%$ \\
\hline Wind Turbines & 2 & $7.1 \%$ \\
\hline HVAC & 2 & $7.1 \%$ \\
\hline Not Mentioned & 2 & $7.1 \%$ \\
\hline
\end{tabular}

\subsection{Multiple Machines Maintenance Planning}

In this subsection, works on maintenance planning on multiple machines are described. These papers are classified according the type of application. 


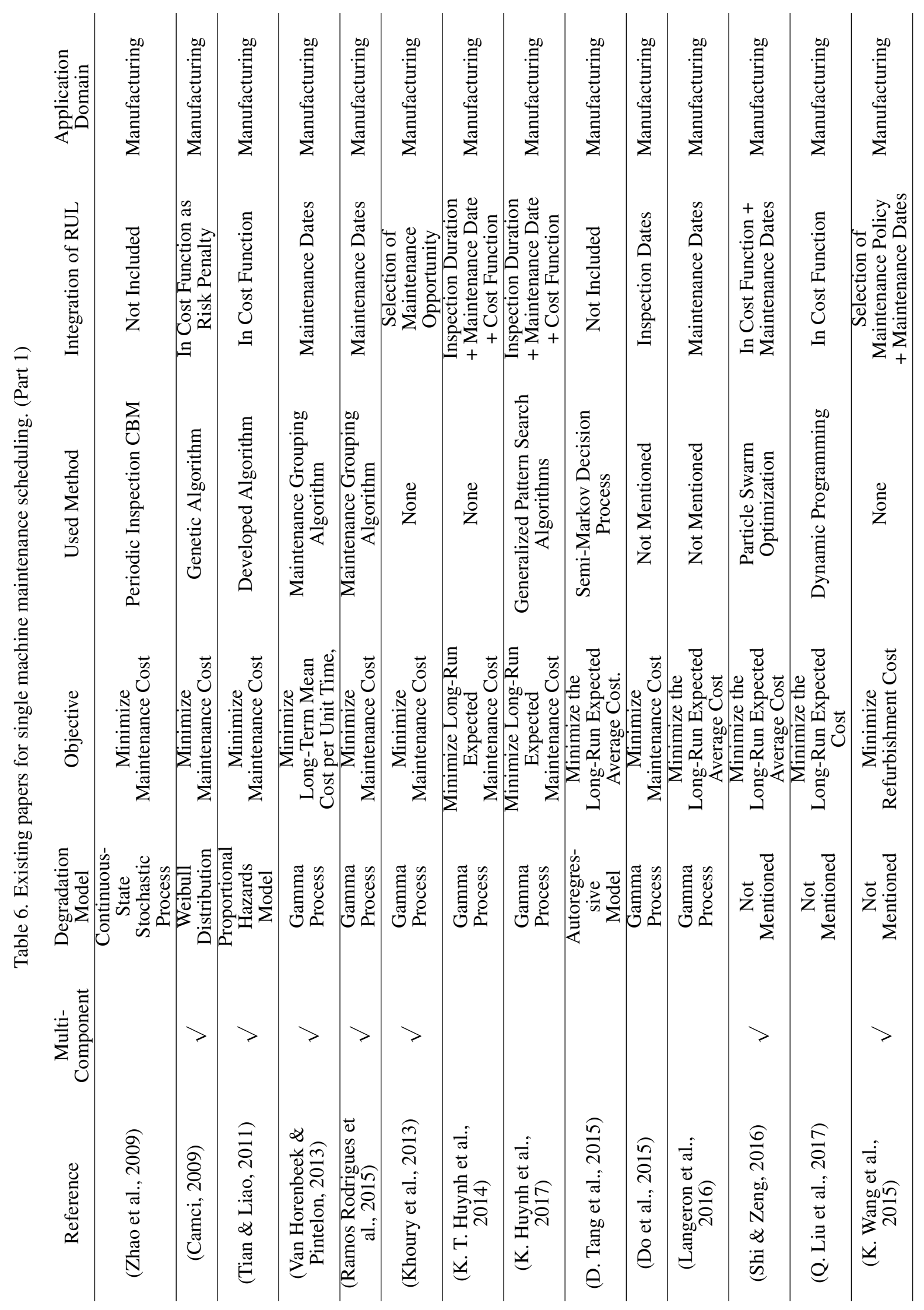




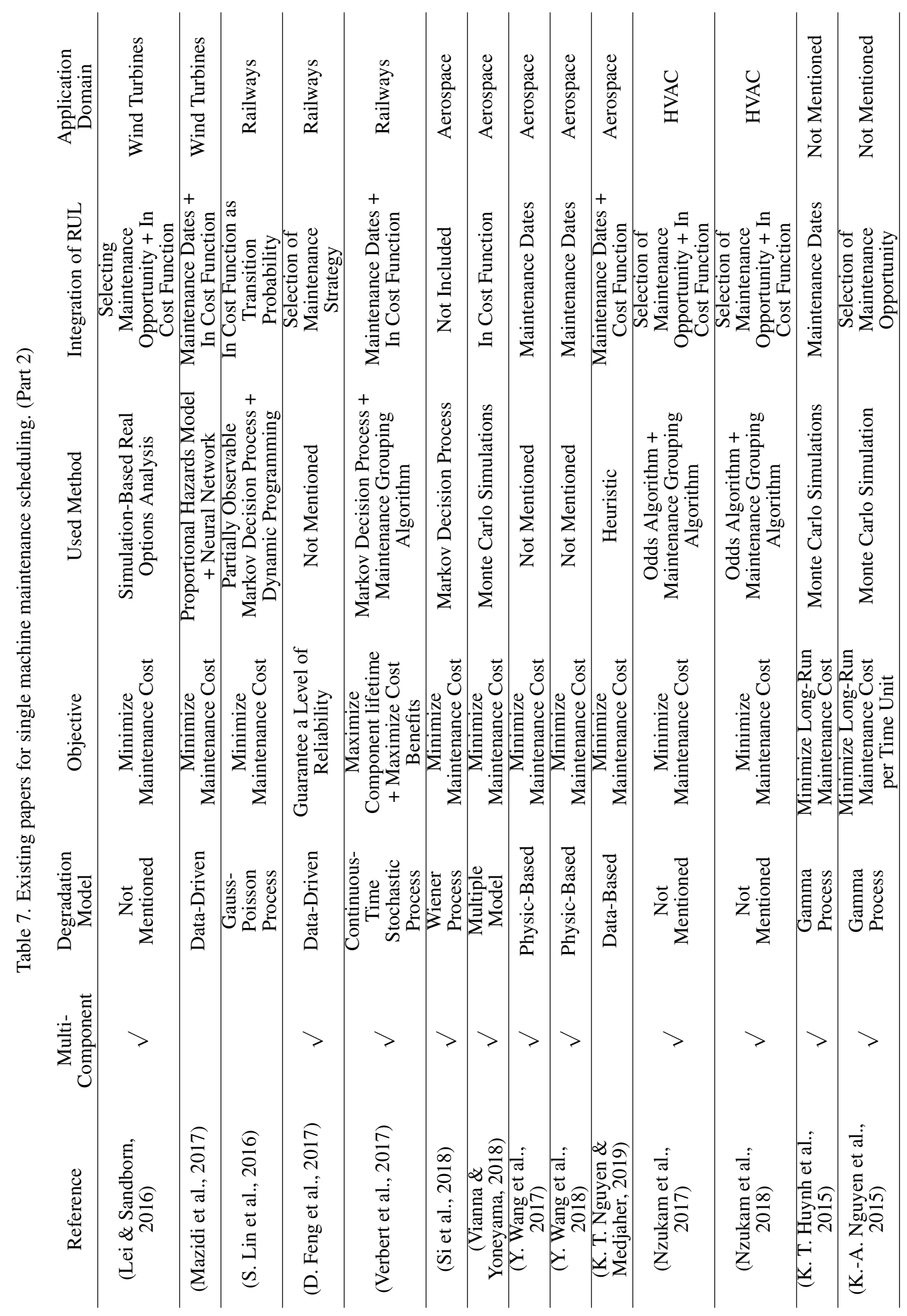




\subsubsection{Production Shop Floor}

Yang et al. proposed in (Yang, Djurdjanovic, \& Ni, 2008) an approach to schedule maintenance activities on manufacturing systems with different typologies of machines. The decisions were based on the forecasted degradation of the machines. Ambani et al. (Ambani, Li, \& Ni, 2009) defined an approach to chose the adequate maintenance policy for multiple serial machines to maximize overall profit. To avoid conflicts caused by the use of common material resources, they proposed two policies for prioritization based on stoppage time and failure sensibility. In (Matyas, Nemeth, Kovacs, \& Glawar, 2017), the authors proposed a framework for maintenance planning in automotive manufacturing industries based on data collected from programmable logic controller (PLCs), quality control services, machine failures and production planning. This framework correlates realtime data to historical failures to predict failure events, health indicators (like RUL) and quality deterioration. The intervention dates are then suggested by the perspective maintenance support system, thus allowing the operators to make the final decision. In (Meraghni, Terrissa, Ayad, Zerhouni, \& Varnier, 2018), Meraghni et al. developed a PHM framework based on Internet-of-Things and cloud computing and cyber physical systems to help maintenance decision-making in big industries. The framework includes maintenance resource allocations, maintenance planning, and traveling cost oriented for maintenance services based on the estimated RUL of the different machines. An example of maintenance technicians traveling cost minimization is given in which the positions of the machines are known and their relative RUL is provided by the prognostic service.

\subsubsection{Wind Farms}

Tian et al. (Tian, Jin, Wu, \& Ding, 2011) addressed the problem of maintenance scheduling for multiple-component wind Turbines in Which a wind turbine is considered a serial connected components (i. e. , if one component fails, the whole system fails). The only dependency considered in this work is economic factors. The policy is based on the failure probabilities that are extracted from the RUL of the wind turbines. Haddad et al. developed, in (Haddad, Peter, \& Pecht, 2011), a new real-option model to optimize the maintenance of offshore turbines based on prognostic indications. The real-option analysis is used to value the maintenance options depending of the RUL of the turbines, thus leading to resolution of the optimization problem of whether to send a maintenance vessel, and if the maintenance team is to be sent, which turbines should be maintained. Lei et al. developed in (Lei \& Sandborn, 2018) a prognostic-based maintenance schedule for wind turbines that are subject to power purchase agreements and prefixed maintenance opportunities. The estimated RUL of the wind turbines is used to define the suitable date of the predictive intervention from the available opportunities. The RUL is periodically estimated to reduce the uncertainty level. At each sampling time, the decision is updated to make sure that the power purchase agreement constraints are satisfied. Wang et al. (P. Wang, Tamilselvan, Twomey, \& Youn, 2013) developed a prognostic informed maintenance decision-making process that takes into consideration the different operational and maintenance costs. In this framework, the degradation level is monitored through periodic inspections. Once it exceeds a predefined threshold, the next maintenance intervention is set based on the estimated RUL by finding a trade-off between operational and maintenance costs.

\subsubsection{Aircraft Fleets}

Goebel et al. (Iyer et al., 2006), proposed a framework for post-prognostic decision-making for airplanes under the constraints of workshops and spare parts availability. Although the work did not include the use of prognostic information, it was a promising approach that was referenced by almost all of these papers. Balaban and Alonso proposed, in (Balaban \& Alonso, 2012), an approach for maintenance decision-making based on prognostic information in the aerospace domain. Rodrigues et al. (Rodrigues et al., 2015) developed an approach to select system components that need to be replaced to lead the system to a desired safety level. The method is based on the combination of system RUL and the system architecture using a fault tree analysis to obtain the failure probability. Cai et al. (Cai, Li, \& Chen, 2016) presented a decision support module for aero-engine maintenance that integrates the RUL of the aero-engine and the repair time distribution to optimize the cost of maintenance of an airplane. Li et al. described, in (Z. Li, Guo, \& Zhou, 2016), a prognostic-based maintenance scheduling for an Air Force fleet. The maintenance scheduling optimizer considers several inputs like the predicted aircraft health indicators, the amount of workload of each maintenance activity, the uncertainties and the remaining flying hours from the already elaborated flying hour program. Lin et al. developed in (L. Lin, Luo, \& Zhong, 2017), a decision support system for aircraft fleets maintenance planning to jointly optimize the fleets availability and its maintenance cost. A novel reliability function is introduced that integrates the real-time load assessment with the current health state of the machine. The RUL is estimated from the reliability function and is used in the decision-making process by penalizing the waste of RUL. In the same context, Feng et al. (Q. Feng, Bi, Zhao, Chen, \& Sun, 2017), proposed a fleet maintenance strategy to minimize overall maintenance costs while meeting the mission risk requirements. The approach is based on dynamic twolevel decision-making that supports a competition and a cooperative game. The RUL of each aircraft is estimated, and the respective failure probability is deduced. According to their RUL and failure probability, the aircrafts are classified 
into two groups: a maintenance group and a standby group. Then a cooperative game takes place between two randomly chosen members of each group to satisfy the mission requirements. The results explain how the combination of the twolevel games provides an optimal solution for the maintenance problem. Luo et al. (Luo \& Lin, 2018) solved the maintenance decision problem for aircraft fleets using a support vector regression method. The RUL of each aircraft is estimated to determine its reliability level. Then it is integrated into the maintenance cost by penalizing the waste of RUL. The proposed approach aims, on the one hand, at minimizing the maintenance cost by minimizing the RUL waste and at maximizing the fleet availability and reliability on the other hand.

\subsubsection{Geographically Distributed Assets}

Jin et al. (Jin et al., 2015) proposed a framework for planning maintenance activities for a geographically distributed manufacturing system. The resulting schedule optimizes the spare parts inventory, resources management and remaining useful life of the machines. Meraghni et al. (Meraghni, Terrissa, Zerhouni, Varnier, \& Ayad, 2016) introduced a framework that provides a schedule of maintenance activities and assigns a maintenance team to each activity for a geographically distributed assets. The methods was tested on cellphone towers in which the PHM system used Internet-ofThings and cloud computing. Aizpuraua et al. (Aizpurua, Catterson, Papadopoulos, Chiacchio, \& D'Urso, 2017) offered new system-level dynamic maintenance planning based on cost-effective grouping of assets. This approach integrates prognostics information, especially the RUL of components, and takes into consideration the dynamic economic and stochastic dependencies between components. The main idea is to schedule predictive maintenance activities for critical components while run-to-failure the uncritical ones.

\subsubsection{Railways}

Camci (Camci, 2014) defined a new variant of the traveling salesman problem that takes into consideration the travel time between machines that need maintenance, that are modeled as cities and the time of their maintenance. This variant was tested on geographically distributed railways switches. This approach aims at minimizing overall cost by scheduling maintenance activities under the constraint of the travel time. In his later work, Camci (Camci, 2015) used the same principle of the traveling maintainer problem (Camci, 2014) with new constraints on the number of working hours of the maintenance team. In this work, Camci defined the frequency of maintenance scheduling and considered schedule changes in case of failures. In (Durazo-Cardenas et al., 2018), the authors designed a maintenance decision support system for railways based on massive data fusion and systems engineering. The proposed system uses collected data to evaluate the degradation levels and the health states of the railways. The pattern of the degradation is then matched with historical data, and if the level of degradation exceeds a threshold, an alarm is generated and maintenance interventions are scheduled according to the operation schedule and the availability of maintenance resources. Villarejo et al. (Villarejo, Johansson, Galar, Sandborn, \& Kumar, 2016), proposed a hybrid model for fault diagnosis, prognostics and maintenance decision-making for railways system. Information is combined from expertise of maintenance workers, rolling stock data, operating conditions and infrastructure data to assess the health of the system and estimate its RUL. The resulting prognostics information is used to plan maintenance interventions to reduce the slowdowns and shutdowns of a rail track.

\subsubsection{Synthesis}

Tables 12 and 13 summarize these papers by specifying if the papers considered multi-components, the used degradation model, the objective of the optimization and the used method, how the RUL of the components/systems are used and the corresponding application domain. One can notice that most of the works do not specify the used degradation model or they assume that the degradation is obtained from historical data (see Table 8). A common assumption in these works is that the RUL of the component and/or the system is supposed to be obtained from a prognostic process.

Table 8. Statistic Summary of Degradation Models for Multi Machine Maintenance Planning

\begin{tabular}{ccc} 
Type of Model & Publications & Percentage \\
\hline Data Driven & 8 & $36 \%$ \\
\hline Physic Based & 1 & $4.5 \%$ \\
\hline Other & 4 & $18 \%$ \\
\hline Not Mentioned & 9 & $40.5 \%$ \\
\hline
\end{tabular}

From Table 9, one can deduce that the objective behind integrating prognostics and health management technologies in the case of multi-machines maintenance scheduling can be either cost-oriented (by minimizing maintenance costs, or maximizing profits) or reliability-oriented (by minimizing failure probabilities and maximizing availability and reliability).

Table 9. Statistic Summary of Objective for Multi Machine Maintenance Planning

\begin{tabular}{ccc} 
Type of Objective & Publications & Percentage \\
\hline Maintenance Cost & 14 & $64 \%$ \\
\hline Profit/Benefit & 3 & $13.5 \%$ \\
\hline Reliability & 4 & $18 \%$ \\
\hline Other & 1 & $4.5 \%$ \\
\hline
\end{tabular}

As these tables show, most works that focused on multimachine systems (55\%), use the remaining useful life as either a penalty on the waste of RUL in the cost function or as a criteria to define the maintenance dates by comparing it 
to a safety threshold (see Table 10).

Table 10. Statistic Summary of RUL Integration for Multi Machine Maintenance Planning

\begin{tabular}{ccc} 
Method of Integration & Publications & Percentage \\
\hline Maintenance Date & 11 & $50 \%$ \\
\hline Cost Function & 12 & $54.5 \%$ \\
\hline Strategy/Opportunity Selection & 3 & $13.5 \%$ \\
\hline
\end{tabular}

Table 11 presents the distribution of the application domain of the found works. One can notice that half of the existing works on multi machine maintenance scheduling are made in the context of aerospace application.

Table 11. Statistic Summary of Application Context for Multi Machine Maintenance Planning

\begin{tabular}{ccc} 
Application Domain & Publications & Percentage \\
\hline Manufacturing & 4 & $18 \%$ \\
\hline Aerospace & 7 & $31.5 \%$ \\
\hline Railways & 4 & $18 \%$ \\
\hline Wind Turbines & 4 & $18 \%$ \\
\hline Geo-Distributed & 3 & $13.5 \%$ \\
\hline
\end{tabular}

\section{Operational Decisions}

In this section, the works that considered operational decisionmaking based on the health information of the systems are studied. As mentioned, operational decisions in the PHM context can be divided into three categories: (i) production planning and mission assignment based on the RUL of the machines, (ii) logistics planning in which the spare parts ordering is optimized to avoid stock shortage and minimize the cost of storage and ordering, and (iii) automatic control decisions in which the control parameters are optimized while considering the health information of the actuator. These categories and their related works are discussed in the next subsections.

\subsection{Production and Mission Planning}

For this type of operational decisions, the authors studied the planning of tasks and/or missions on different configurations of systems. There are two levels for this problem. Some authors considered the mission or tasks assignment by choosing which machine to perform a given mission based on predictive information either of the machine (e. g. degradation level or RUL) or of the mission (e. g. the estimated effects of performing such task on the given system). While other authors considered the scheduling and the sequencing of missions and tasks on the given system also while considering prognostic information. The works proposed different resolution methods to solve the problem while optimizing an objective function under several constraints imposed by the considered application.

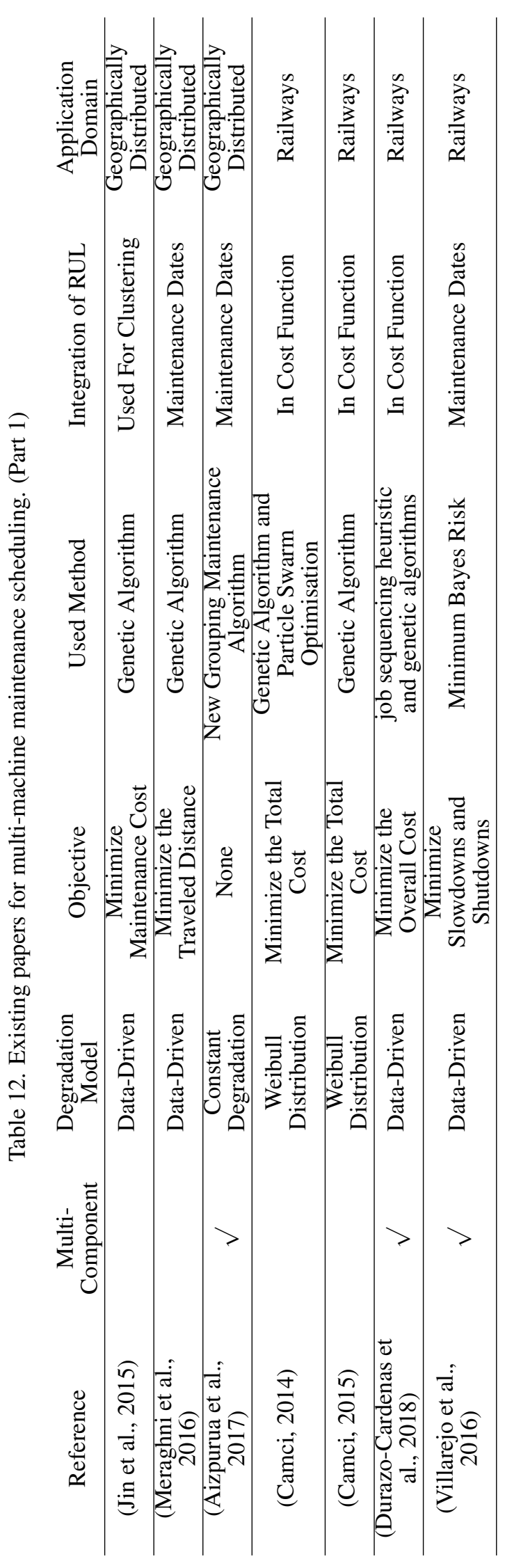




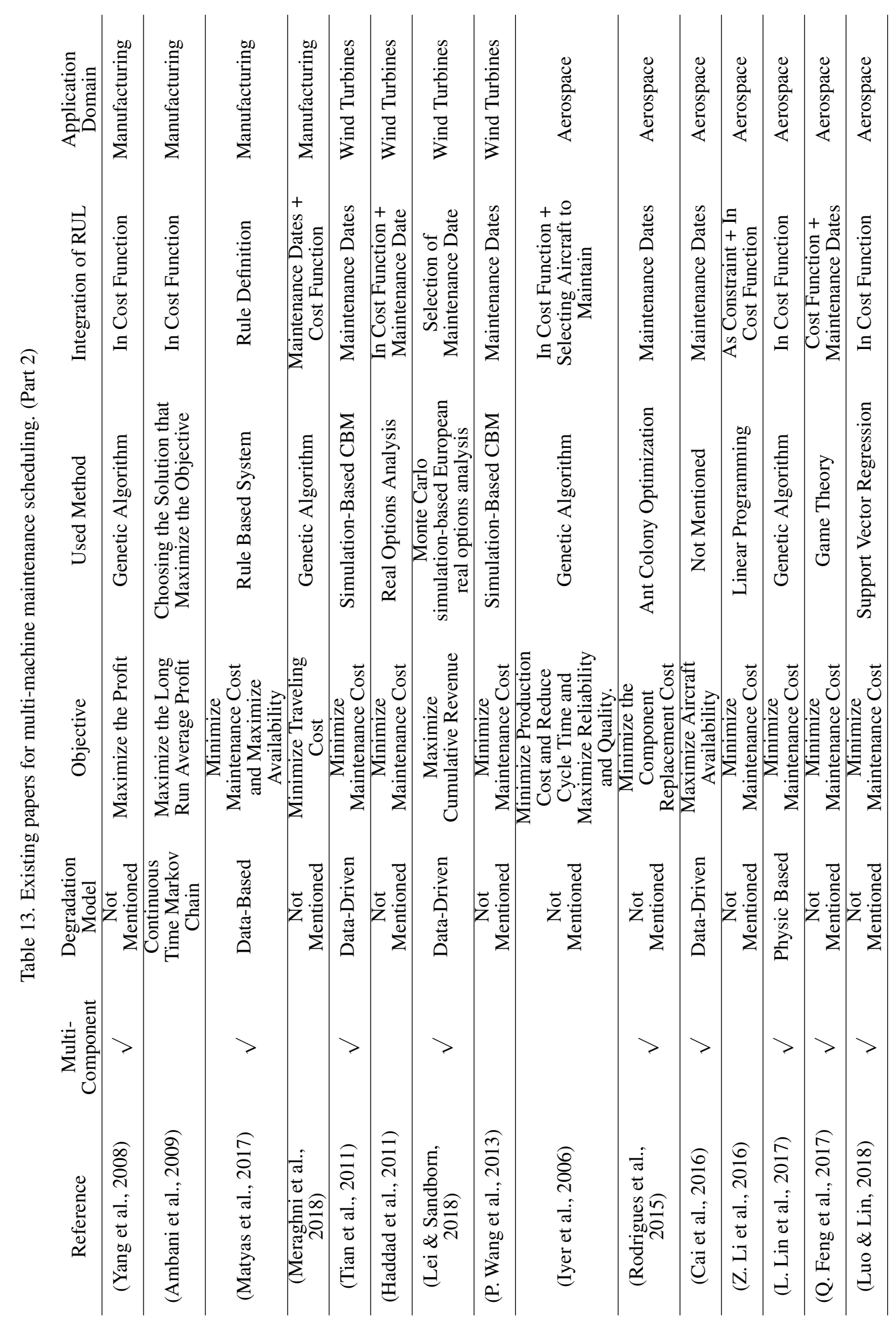


Tang et al. (L. Tang, Hettler, Zhang, \& DeCastro, 2011) presented a real-time autonomous vehicle PHM and contingency management application framework. The framework has the ability to proactively and autonomously adapt to changes in the health states of the vehicle while achieving all acceptable subsets of the mission objective. This is done by low and medium control levels (by controller configuration) and highlevel mission re-planning and optimization while integrating prognostics indicators like the end-of-charge and RUL. The authors focused on mission re-planning so that when a fault occurs, the system periodically estimates the RUL values, which are then used as new constraints or additional elements in the cost function of the mission planning algorithm. Cholette et al. (Cholette, Celen, Djurdjanovic, \& Rasberry, 2013) implemented prognostic information in the production scheduling of semi-conductors. The solution proposed the scheduling of products between different machines by rerouting products from a degraded machine to a less degraded one to increase yield and the probability of mission success. To satisfy a production demand and maximize the production horizon, Herr et al. proposed different heuristics in (Herr, Nicod, \& Varnier, 2014). The algorithm consists of choosing $q$ of the $m$ parallel machines that are capable of performing independent and identical tasks. Their adequate running profiles are also determined to fulfill the mission (production level). Zhang et al. developed in (Zhang, Tang, Decastro, \& Goebel, 2011) and (Zhang, Tang, DeCastro, Roemer, \& Goebel, 2014) a mission-planning algorithm for an autonomous vehicle, that enhanced prognostics information in the decision-making process. The algorithm is based on a $D^{*}$ search algorithm to find a suitable road on an unknown map. The best road was chosen gradually as the rover moved. Different objectives were considered in the objective function, including RUL optimization. Medeiros et al. (de Medeiros, Rodrigues, Santos, Shiguemori, \& Júnior, 2014) presented a task assignment algorithm that considers health monitoring information obtained by the distribution of components RUL and the system fault tree representation. The proposed receding horizon task assignment (RHTA) algorithm aims at increasing the probability of the tasks' success. Rodrigues $e t$ al. (Rodrigues, Gomes, \& Alcântara, 2018) proposed a resolution of the task assignment problem for unmanned aerial vehicle (UAV) subject to the remaining useful life of the vehicles. The modified Receding Horizon Task Assignment algorithm uses a rejection list to reduce the number of mission combinations. At each step of computation, the obtained sub problem (after applying the reject list) is considered a multiple choice multidimensional knapsack problem. Chretien et al. (Chrétien, Herr, Nicod, \& Varnier, 2016) proposed a post-prognostic decision framework for scheduling multistack fuel cell systems under service constraints. The problem considers the fuel cells parallel independent machines. It was solved using two convex resolution methods, mirror prox algorithm and adaptive lasso algorithm. Herr et al. de- veloped a framework in (Herr, Nicod, Varnier, Jardin, et al., 2017) for managing fuel cell stacks to satisfy a load demand for as long as possible. The problem combined production decisions by selecting the power output of each fuel cell with the task assignment decisions by choosing which fuel cell stacks to run. The problem was then solved using mixed integer linear programming that integrated prognostics information about the health state of fuel cell which are subject to wear and tear behaviors. Zuo et al. (Zuo, Cadet, Li, Bérenguer, $\&$ Outbib, 2020) proposed a heuristic that manages the load distribution for a two-stacks fuel cell system to maximize the service time. The authors considered that the fuel cells' degradation could be described by a Gamma process that depends on the load of the system. Skima et al. (Skima, Varnier, Dedu, Medjaher, \& Bourgeois, 2017) applied postprognostics decision-making for a conveying surface made of micro-electro-mechanical systems. The problem in this paper is choosing the proper path to transport micro-objects from a source block to a destination block in a way that maintains the system's best conditions for as long as possible. To improve the conveying surface performance, the proposed algorithm combined the use of already obtained prognostics information to maximize the lifetime of the surface and the use of travel time of the objects to optimize the utilization of the conveying surface. Tamssaouet et al. (Tamssaouet, Nguyen, \& Medjaher, 2019) proposed to maximize the RUL of a rail vehicle through the optimization of the mission's parameters. The authors considered that the rail vehicle is composed of several components and they modeled the overall health of the vehicle through an inoperability input-output model. They used genetic algorithm to optimize a railway vehicle's mission profile parameters (e. g. speed, load, etc) to maximize the RUL of the considered system.

Table 14 papers, that treated production or mission planning in the context of PHM. Most of these works are built on the assumption of having a prognostic module that provides the RUL of the machines and/or their components. This assumed prognostic module also provides the health state requirement of certain production jobs or mission and how much they affect the systems' RUL. For these reasons, most of these works do not specify the degradation model ( $45.5 \%$ of found papers) or the method used to estimate the RUL. Instead, they focus on the decision process and its optimization procedure. The objectives of these papers can be classified into three categories: (i) time-oriented objective (in which the focus is on extending the planning horizon $27 \%$ or the life time of the system $18 \%$. or minimize the mission duration $18 \%$ ); (ii) cost-oriented objective by maximizing the benefits $9 \%$; or (iii) reliability-oriented by minimizing failure risk $28 \%$. Various optimization algorithms are used in the decisionmaking process: local search algorithms, linear programming, path-finding methods and task assignment methods. The integration of the RUL into the decision-making process 
for production and mission planning is more dynamic than for maintenance decisions. This can be observed through the use of RUL not only as a penalty in the cost function or as a constraint but also as a decision variable for selecting the suitable path ( $63.5 \%$ of the found works) or by making a reject list in the case of mission assignment (18\% of the found works). Another important point is that most of these decision-making processes are based on one RUL estimation for the whole decision-making process. Thus, the dynamics of RUL estimation are not considered in Table 14.

\subsection{Logistics Planning}

The authors studied the planning of logistic movements on different configurations of systems. They considered the ordering of spare parts and inventory management while considering the predictive information of the operating system. The works proposed resolution methods to solve the problem while optimizing an objective function under several constraints imposed by the considered application.

Li and Ryan (R. Li \& Ryan, 2011) modeled the deterioration of components with a Wiener process and used a Bayesian approach to estimate the distribution of the RUL. The predicted end-of-life is updated periodically by integrating the new available system data through condition monitoring. The estimated RULs define the demand for spare parts in the future. This demand is then used to order the spare parts.

To meet the delivery commitment of at least possible cost, Julka et al. (Julka et al., 2011) integrated the use of prognostic information in the discrete event logistics systems (DELS). The RUL was integrated into the D-SIMPAIR system to optimize the inventory and movement of spare parts by predicting the system failure date and moving the spare parts to a location where maintenance will be conducted. Based on the same context, Cui et al. (Cui, Shi, \& Wang, 2015), proposed a modification of the DELS. The approach is based on a two step RUL estimation in which the first estimated RUL is used to define the logistics movements according to the maintenance events activities, while the second estimated RUL is used to calibrate the fault occurrence time. The obtained system aims at arranging the spare allocations ahead of the fault occurrence, which largely reduces the logistic delays. Lin et al. (X. Lin, Basten, Kranenburg, \& van Houtum, 2017) considered the case of a group of identical machines that each contained one critical component. The degradation of these components is monitored and the remaining useful life distribution of each part is estimated. The RULs are updated at each inspection period, and the results are used to determine the distribution of the demand for spare parts in the upcoming periods while considering a non zero lead time for spare parts delivery.

Usually, logistic decision-making is highly related to maintenance decisions. To our knowledge, only four works treated logistics decisions alone. These works were described earlier and are summarised in Table 15. One can note that stochastic processes are commonly used for the logistics decisionmaking, to implement the uncertainties in estimations. Although all these works have a common cost-oriented objective to minimize the inventory and logistics costs, various methods are used to achieve that. As for the RUL, it is estimated with different dynamics from only one time to a periodic estimation, and it is used mainly to define the ordering dates for the spare parts, taking into consideration the leadtime to deliver the orders. Most of the works that treat logistic decisions, are in aerospace or heavy manufacturing applications, in which the spare parts are large and thus require a large storage facilities. Thus the cost of inventory is expensive, and the storage of multiple spare parts can be difficult.

\subsection{Automatic Control}

In this subsection, the authors considered smaller parts of the system (i. e. actuators). They proposed enhancement of the control loop by considering the health state of the component. Different methods are used to integrate the predictive information in the control loop to maximize the actuator life spin or its' reliability level.

Bogdanov et al. (Bogdanov, Chiu, Gokdere, \& Vian, 2007), designed a linear-quadratic regulator $(\mathbf{L Q R})$ controller that has a single scalar as a parameter that establishes a tradeoff between performance, the desired lifetime and the control power. The actuator in the paper is supposed to be under stochastic external load that affects its lifetime. A constraint is made on the desired lifetime, and the main work is to optimize the parameter of the LQR controller. Brown et al. (Brown et al., 2009) presented a methodology for designing a fault-tolerant controller that re-configures the actuators' control activity using prognostics information by trading off performance and control power. Composed of electromechanical actuators, the system is supposed to complete a critical mission within a time window. This defines the limits of the performance of the actuator and thus the controller has to be able to reconfigure the control activity to secure an acceptable performance level that satisfies the mission need. Later, Brown et al. (Brown \& Vachtsevanos, n.d.) presented an approach in elaborating a prognostic-based reconfigurable control for electro-mechanical actuators. The framework is based on a PHM module parallel to the controller. The main idea consists of comparing the RUL to a desired value. If the system can secure the desired lifetime, then no action has to be taken. Otherwise, a reconfiguration is triggered by allowing new acceptable minimum and maximum tracking errors that will change the cost function of the model predictive controller (MPC). Consequently, the control signal will change and some set-point adjustments will be obtained. Bole et al. (Bole, Tang, Goebel, \& Vachtsevanos, 2011) developed a prognostic-based controller that will adaptively allocate the 


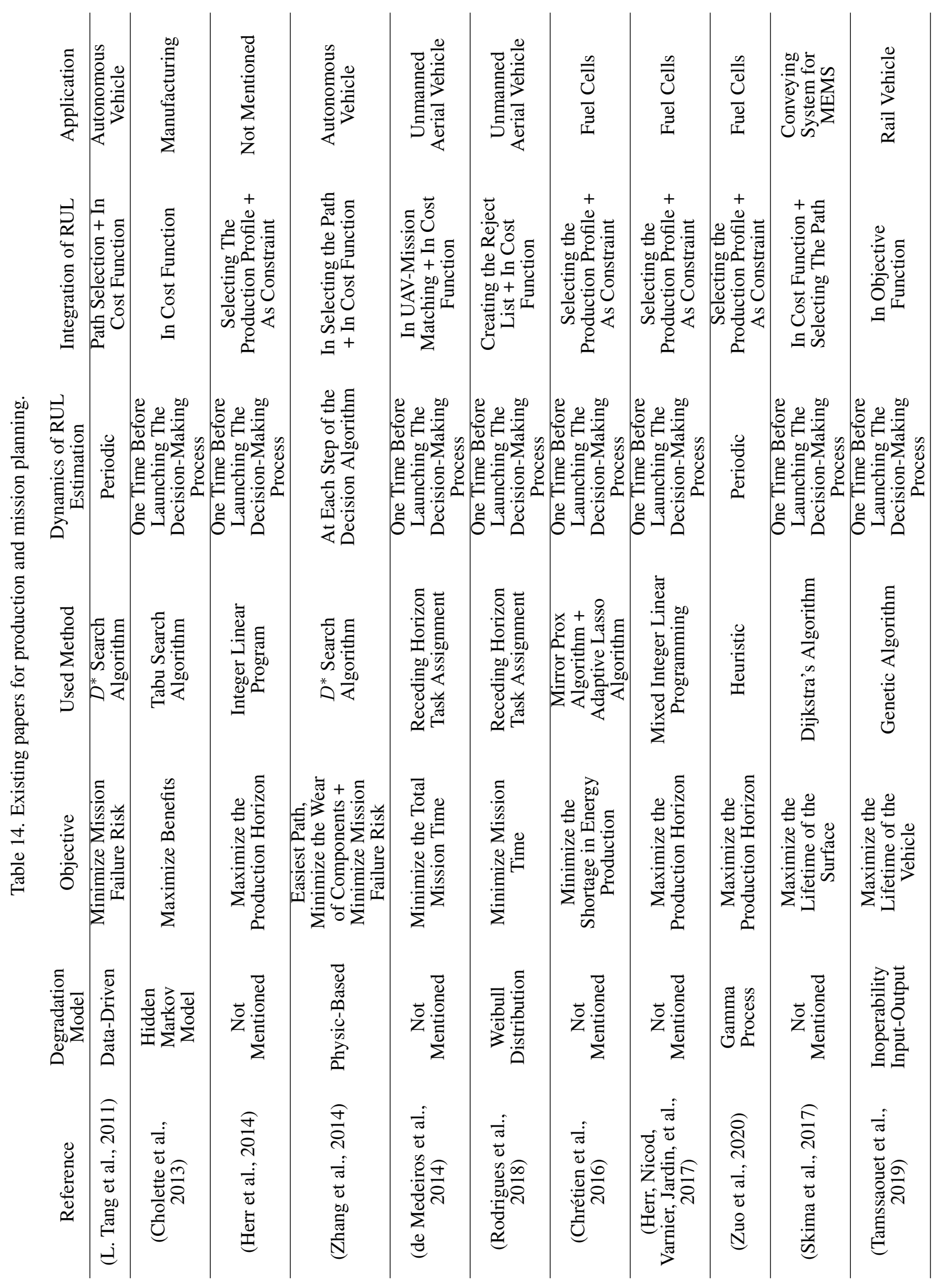




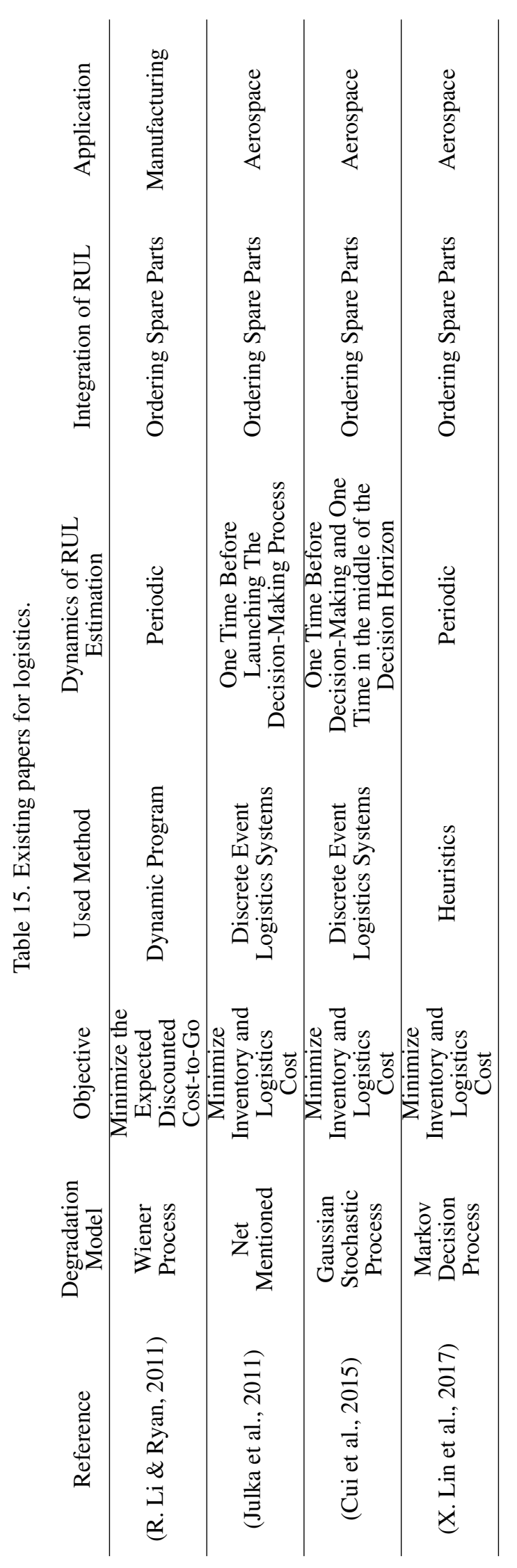

actuator's load to optimize a risk metric based on the uncertain RUL. The prognostic-based fault-adaptive controller finds a trade-off between minimizing the failure risks and the system's overall performance "without becoming overly conservative". Pereira et al. (Pereira et al., 2010) developed a predictive control based on the prognostics and health monitoring of the actuators. The main idea is to determine the remaining allowed degradation until the end of life of the system and then distribute it uniformly on the remaining time until the next scheduled maintenance. This approach has been tested on systems with redundant actuators. In the same context, Langeron et al. (Langeron et al., 2013), proposed a LQR controller, in which the matrices $Q$ and $R$ are modified with the evolution of the system degradation. Nguyen et al. (D. N. Nguyen, Dieulle, \& Grall, 2014) improved the RUL estimation process of the closed loop systems. The output RUL is then used to configure an adaptive controller. Vieira et al. (Vieira, Galvão, \& Yoneyama, 2015) proved the existing relationship between the degradation of the actuator and the loss of its effectiveness. They also used the results in the configuration of the LQR controller parameters. The results obtained in (Vieira et al., 2015), were used by Langeron et al. in (Langeron, Grall, \& Barros, 2015) to model a closed loop system that can fully implement the control law regardless of its degradation. The system is controlled by a LQR in which its parameters are adapted using the degradation level of the actuators through its RUL. Grosso et al. (Grosso, OcampoMartinez, \& Puig, 2016) proposed an improved reliabilitybased economic model predictive control strategy that is similar to the predictive control proposed by Pereira et al. in (Pereira et al., 2010). The idea consists of computing and distributing the remaining allowed degradation level on the remaining time to maintenance but with an additive dynamic stock policy in the optimization problem. The system has to guarantee the spatial and temporal re-allocation of water resources under demand uncertainty. This strategy was used on a centralized control for distributed actuators of the water network distribution of Barcelona. Niu and Liu (Niu \& Liu, 2018) used condition monitoring to estimate the current degradation of the component. Then, the authors estimated the evolution of the component's fatigue and damage under different control laws. Finally, they extracted the control law that stabilise the evolution of fatigue and damage to extend the remaining useful life of the component.

Table 17 summarizes these works. Most aim to compute new control set points or controllers' parameters taking into consideration the health state of the actuator. Since the application can be considered a low-level control process, the degradation models are most likely based on the physics of the degradation of the actuator. In some cases, when the physics of degradation are complex the authors tend to use stochastic processes to model the deterioration of the actuator (see Table 16). 
Table 16. Statistic Summary of Degradation Models for Control Decisions

\begin{tabular}{ccc} 
Type of Model & Publications & Percentage \\
\hline Physic Based & 5 & $45.5 \%$ \\
\hline Stochastic Process & 4 & $36.5 \%$ \\
\hline Not Mentioned & 2 & $18 \%$ \\
\hline
\end{tabular}

As Table 17 shows, almost all the works aim to extend the lifetime of the actuator $(64 \%)$ by balancing the performance and the control power. Automatic control loops are characterized with high speed feedback dynamic. Thus, the estimation of RUL needs to follow the same high speed that is guaranteed by the chosen degradation model and the estimation techniques. Consequently, the decision-making process needs to match the speed of the feedback dynamic and the speed of changes in the actuators' health state. Therefore, one can note that works that investigate automatic control usually avoid classic optimization algorithms, which can take a long time to compute and find solutions, and to use analytical methods instead. As for the RUL, it is most commonly used as a constraint in the computation of new control settings or included in the function that defines the new settings or in the cost function itself.

\section{MiXed DeCisions}

Mixed decision-making in this work refers to works that jointly optimize operational and maintenance decisions. As noted in the previous section, operational decisions are classified into three subcategories. Therefore, mixed decisions can also be classified into three subcategories: (i) mission and production planning jointly with maintenance, (ii) automatic control with maintenance scheduling, and (iii) logistics or spare parts ordering jointly with maintenance planning. These subcategories are described in the following subsections. In addition, some works do not present a specific type of mixed decision-making, but they present frameworks to mixed decision-making on an enterprise level. These works are also discussed in this section.

\subsection{Mission or Production Planning Jointly with Main- tenance}

Medeiros et al. (De Medeiros, Rodrigues, Kern, Dos Santos, \& Shiguemori, 2015) proposed a joint task assignment and maintenance scheduling for unmanned aerial vehicle (UAVs). A fault tree is used to determine the failure probability of the system based on the components RUL, while a RHTA algorithm with PHM information is responsible for the task assignments. Herr et al. in (Herr, Nicod, Varnier, Zerhouni, et al., 2017) jointly optimized the rolling stock assignment and maintenance scheduling of trains. The main idea is to assign the suitable train to each trip and minimize the waste of useful life of components while avoiding their failure by taking into consideration the predefined train timetables and prognostics information. Niknam et al. (Niknam, Kobza, \& Hines, 2015) developed a method to optimize the decisionmaking to control the system and schedule its maintenance. The method was tested on a variable speed wind turbine by controlling its speed and planing the maintenance actions. Pan et al. (Pan, Liao, \& Xi, 2012) proposed a mathematical programming formulation to solve the joint problem of production and maintenance scheduling on a single manufacturing machine. In this work, the RUL of the system is estimated and then remaining maintenance life (RML) is deduced by respecting a minimal reliability level. Once the RML is obtained, production and predictive maintenance are scheduled in a way that minimize the total tardiness of jobs and respects the RML constraint. Another contribution presented in this paper is the influence of the age of the machine on its degradation dynamic. It is assumed that after a certain number of maintenance interventions, the machine deteriorates more quickly. Wang (W. Wang, 2014) used a prognostic information based method to schedule the suitable task and preventive maintenance on a stochastic degrading single manufacturing machine. The method aims at maximizing the long-term expected profit per unit time. Fitouri et al. (Fitouri, Fnaiech, Varnier, Fnaiech, \& Zerhouni, 2016) proposed a heuristic to solve the problem of job shop production and predictive maintenance scheduling. The approach aims at minimizing the Makespan and the total cost of maintenance based on prognostic information. In their approach the RUL of each machine depends on the task in progress. Ladj et al. (Ladj, Varnier, Tayeb, \& Zerhouni, 2017) solved the integrated production and maintenance scheduling for a multi-functional single machine, to minimize the total maintenance cost, in which each job is characterized by a degradation level. The solution contains two methods mixed-integer linear programming and pro-genetic algorithms, and took into consideration the consumption of each job in terms of degradation. Later, the same authors in (Ladj, Benbouzid-Si Tayeb, Varnier, Dridi, \& Selmane, 2017) proposed a method for jointly planning production and maintenance activities based on prognostic information in the case of permutation flow-shop scheduling problems. The predicted RUL and degradation values are associated with each machine when processing each kind of job. Uncertainties in this work are modeled through the use of fuzzy logic to model the RUL distributions. The main objective is to find the best sequencing of jobs to optimize the maximum completion time (Makespan) and the maintenance cost simultaneously. Desforges et al. (Desforges, Diévart, \& Archimède, 2017) presented a generic equation to evaluate the system's capacity to satisfy the requirements of future production planning. The object oriented Bayesian network based equation includes different levels of system modeling: a functional model, a structural model and a behavior model. The prognostics of different components are used with the 


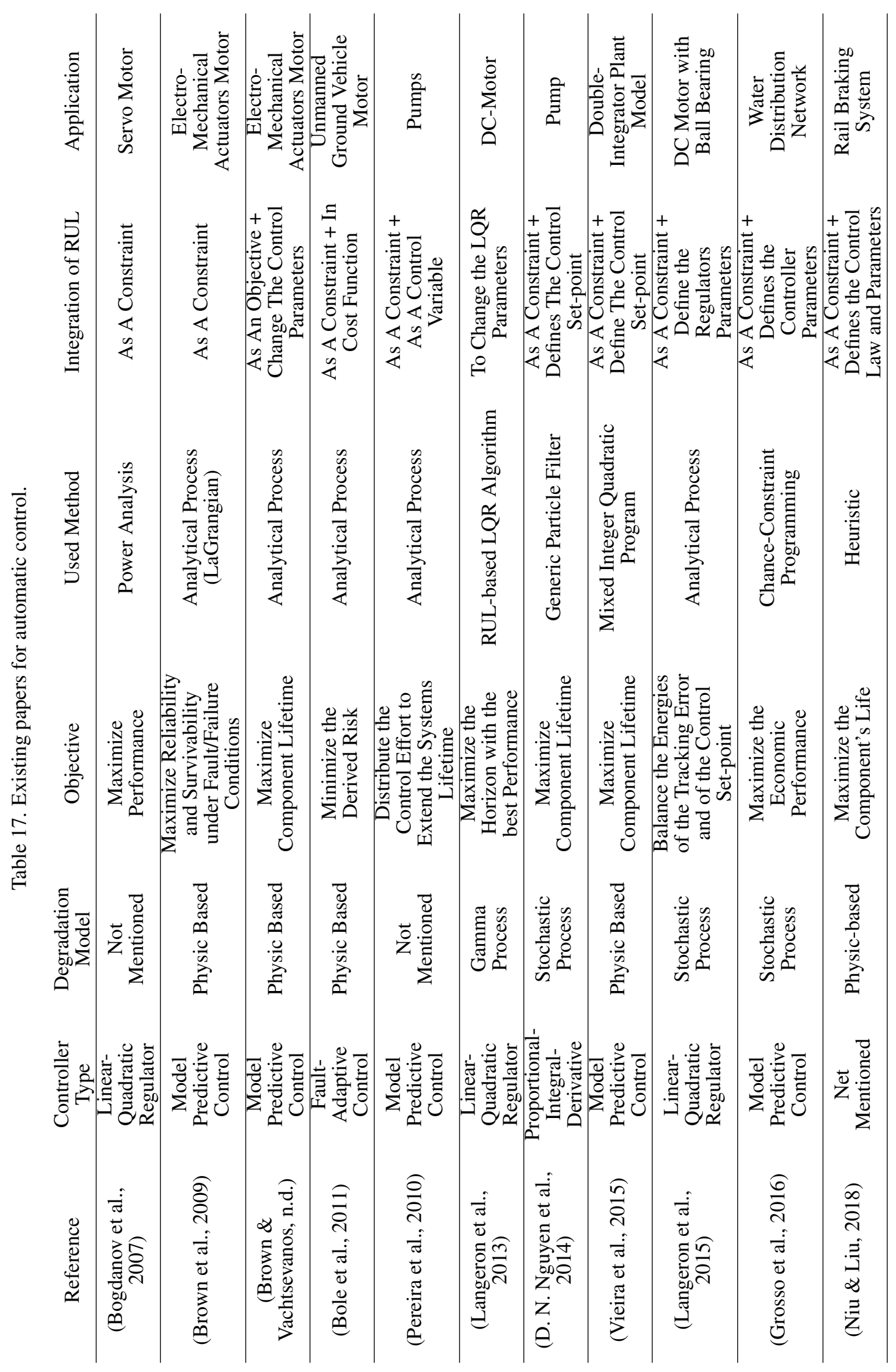


future load to assess the system capacity at fulfilling its future plans while suggesting which components to maintain to guarantee a predefined minimal level of reliability. Cheng et al. (Cheng, Zhou, \& Li, 2018) proposed a method that optimizes the production, quality control and condition-based maintenance for a stochastic deteriorating manufacturing system. A periodic inspection CBM policy is used to define imperfect maintenance actions. The degradation level of the component is combined with the quality control inspection data to define the maintenance dates. While the system is producing, the degradation of its components is monitored, and the ratios of defectives from the quality control inspections are compared respectively to a preventive threshold and a quality threshold. The excess of degradation level or the ratio defect causes the system to stop for preventive or corrective maintenance. Bencheikh et al. (Bencheikh, Letouzey, \& Desforges, 2018) proposed a multi-agent-based system to solve the problem of jointly scheduling production and maintenance on a multi-purpose multi-machine workshop. Each machine is capable of different functions, and the scheduling of the production activities and maintenance interventions takes into consideration the current and future health state of the machine. Bougacha et al. (Bougacha, Varnier, Zerhouni, \& Hajri-Gabouj, 2018) addressed the problem of scheduling jointly maintenance and production tasks on a single multi-purpose machine. In this paper, the scheduling of production jobs is based on the machine capacity in satisfying the task requirements and guaranteeing a certain level of reliability. Whenever the estimated machine degradation reaches a predefined threshold, a preventive maintenance intervention is planned. The main idea is to find the suitable trade-off between producing with different production speeds and maintaining the system components to maximize the total profit. In (Q. Liu, Dong, Chen, Lv, \& Ye, 2019), the health state and the RUL of the machine are used to schedule jointly production activities and maintenance actions to improve the machine utilization, decrease the failure rate, and minimize the total costs, including the production cost, maintenance cost, downtime cost and tardiness cost. In this work, Liu et al. used a Weibull distribution to model the degradation of the machine. Benaggoune et al. (Benaggoune, Meraghni, Ma, Mouss, \& Zerhouni, 2020) studied the effects of RUL uncertainties on the resolution of the joint problem of maintenance scheduling and production planning for a multi purpose single machine. The authors used a particle swarm optimization algorithm to search for the best schedule of production jobs and maintenance interventions that minimizes the maintenance cost.

Table 18 summarizes these papers. One can note the big variety in the degradation models used for these works. Some works did not focus on the degradation model of the system or the components and just assumed that each job has a failure probability related to the system health. Most likely these failure probabilities are not explained and the authors did not show how they obtained such information. Others used deterministic models by supposing that the degradation of the system can be described through a simple function (exponential as in (Bougacha et al., 2018)) or that each job/task is associated with a predetermined level of degradation called task severity in this paper. The objectives of these works can be gathered under three categories: (i) production-oriented objectives, which can be found in the literature of classic production scheduling problems like minimizing the tardiness or the Makespan; (ii) maintenance-oriented objective, by trying to minimize maintenance cost or minimizing the waste of the useful life; and (iii) mixed objectives, that includes both maintenance and production objectives indirectly like maximizing the profits, or multi-objective problems in which there are two objectives, like in the case of (Fitouri et al., 2016). Thus, different kinds of optimization method have been used to solve the problems. Finally, RUL is used differently in these works, mostly to define the capacity of achieving a task, to define the maintenance date or as a constraint by comparing it to a threshold. In other works, the RUL is directly used in the cost function by penalizing the waste of useful life or by implementing the failure risk.

\subsection{Automatic Control Jointly with Maintenance}

Langeron et al. (Langeron, Grall, \& Barros, 2017), presented an approach that defines operational decision-making by changing the matrix $Q$ and $R$ of the LQR controller and scheduling the maintenance actions based on the RUL of the system. This approach was tested on a DC motor by varying its speed and planning the replacement of its bearing. Jain et al. (Jain \& Lad, 2017) established a relationship between product quality and the tool degradation level. Based on this relationship, they designed a dynamic policy for optimizing process quality control and preventive maintenance. The method integrates real-time RUL estimations. The production process starts with its optimal pre-established parameters. Then, at each sampling time, the RUL of the tool and its health status are estimated, upon which the parameters of the production process are re-adapted to the RUL. Maintenance interventions are scheduled in parallel in a way that balances the cost of waste of remaining life and the cost of loss of quality computed through the ratio of defects. Griffith et al. (Griffith, Yoder, Resor, White, \& Paquette, 2014) proposed a structural health and prognostic management system to enhance the use of prognostic information like health indicators and RUL in the maintenance decision-making and prognostic control jointly. The health information in this case are used to define the operation controls and the maintenance intervention scheduling.

Niu and Jiang (Niu \& Jiang, 2017) proposed a novel methodology to optimize the maintenance of braking system in rail operations. First, they compute the offline braking force pro- 


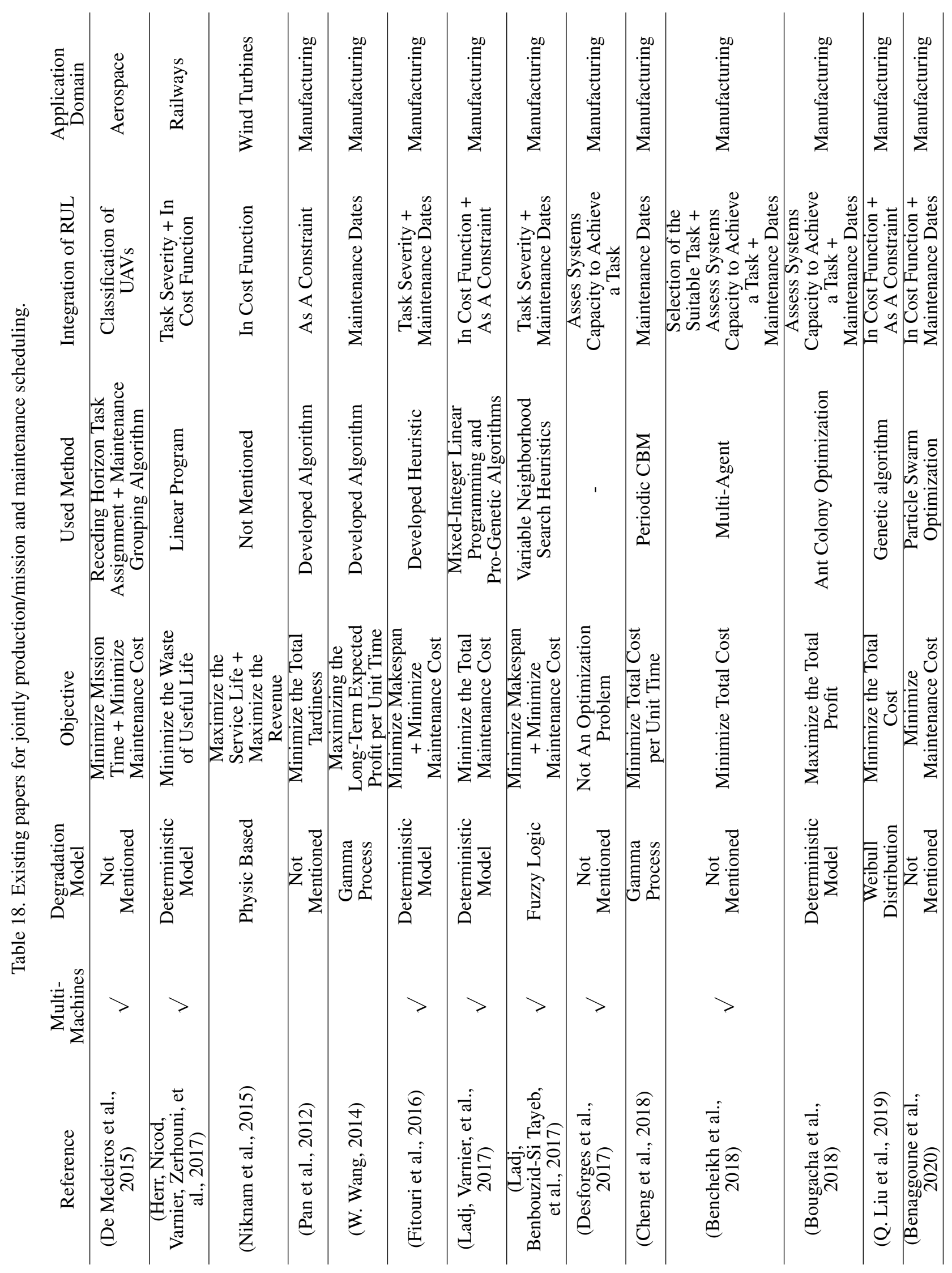


file based on the condition previous experiences. Then, they compute the online braking force profile that relieves the wear of the brake. Finally, on a system level, they plan maintenance actions on the brakes to minimize the cost of maintenance per $\mathrm{km}$. extends the life of the brakes.

\subsection{Logistics Jointly with Maintenance}

Wang et al. in (Z.-Q. Wang, Wang, Hu, Si, \& Zhang, 2015) presented a RUL-based spare parts ordering and predictive maintenance scheduling for a stochastical deteriorating nonrepairable critical system. Wiener process was used to model the degradation of the system, in which its parameters are estimated from the real-time condition monitoring data. The obtained Wiener process is used to estimate the RUL distribution that are used to update ordering and the maintenance schedule. This method aims at minimizing the expected cost rate over an infinite time horizon. Wang et al. in (Z. Wang, Hu, Wang, Kong, \& Zhang, 2015) proposed a prognosticbased spare part ordering and maintenance intervention for an aerospace system with a random lead time. The dynamic method consists of periodically collecting new monitoring data related to the condition of the system. Based on these data, the degradation model parameters is estimated, and the RUL is predicted. The RUL is then used to feed the optimization of the ordering date and the maintenance intervention schedule. Wang et al. (C. Wang, Xu, Wang, \& Zhang, 2018) proposed a multi-spare ordering policy for complex systems with multiple continuously deteriorating components. The method consists of computing the overall reliability of the system from the components' reliability. To guarantee a predefined level of reliability of the system, some components are chosen to be maintained or replaced and their respective spare parts orders are issued to minimize the overall cost of maintenance, ordering and storage. Cai et al. (Cai, Yin, Zhang, \& Chen, 2017) studied a joint optimization of maintenance and spare part inventory with appointment policy. The authors combined the classic CBM approach with periodic inspection of components with the estimation of the RUL to appoint spare parts to the component with an estimated RUL below a specific threshold. Once the spare part inventory is lower than a predefined security level, the spare parts orders are placed. Later, the authors of (Cai, Li, \& Chen, 2017) considered the same approach but this time instead of using a periodic inspection policy, the inspection dates are defined according to the degradation phase of the components. In this work, the inventory is kept empty for a certain duration under the assumption that during the first part of its lifetime the aircraft does not need any maintenance interventions. Afterwards, the demand for spare parts grows gradually and stabilizes at a certain level. At this point, the inventory will be monitored and compared to a security level to place the orders.

Chen et al. (Chen, Xu, \& Xiao, 2016) introduced a method to predict RUL of an individual component when the degradation cannot be reflected by historical data in totality. The estimated RUL is formulated into failure probability function, upon which maintenance interventions and spare part ordering are optimized jointly. Bousdekis et al. (Bousdekis, Papageorgiou, Magoutas, Apostolou, \& Mentzas, 2017) developed a proactive decision-making framework for conditionbased maintenance. This framework optimizes jointly the maintenance and the spare parts inventory based on prognostics information. The module defines the maintenance activity time by minimizing the long-term maintenance cost and the best time to order spare parts by minimizing the longterm inventory cost. Liu et al. (X. Liu, Yang, Pei, Liao, $\&$ Pohl, 2019) developed a heuristic based on a Markov decision process to solve the problem of maintenance replacements and spare parts ordering in the case of single manufacturing machine. The Markov decision process integrated health information of the system component when this information is directly obtained from the degradation model. The method aims at maximizing the net revenue of the workshop.

Moghaddass et al. (Moghaddass \& Ertekin, 2018) proposed a dynamic decision policy to jointly optimize ordering and replacement dates for a single-unit inventory system. The proposed method consists of periodically collecting and observing data related to the system operations and then determining whether to start the setup of a maintenance intervention on one level. On the second level, the method is used to determine when a maintenance activity should take place. At each inspection, the current degradation level and the system health state are assessed and then the failure probability until the next inspection date is computed. This information is integrated in the decision-making process to generate warnings and schedule maintenance to minimize the long-run expected cost per unit of time.

Table 19 summarizes these works. First, almost all the works that dealt with spare part ordering and maintenance planning used stochastic process for the degradation model. Works in the aerospace domain used the Wiener process. The objectives of these works are always cost-oriented, in which either the cost are to be minimized or revenues and benefits to be maximized. As for the method used to solve the optimization problem, mostly each of these works developed its own heuristics with the exception of two that used genetic algorithms with Monte Carlo simulations. All these works used the prognostic information in the same way, i. e. , to define the maintenance and the spare part ordering dates. Actually, the works of each domain are quite similar, and the only difference is the proposed heuristics. Sadly, neither of these works proposes a comparison between the results they obtained and the results obtained by other authors. 


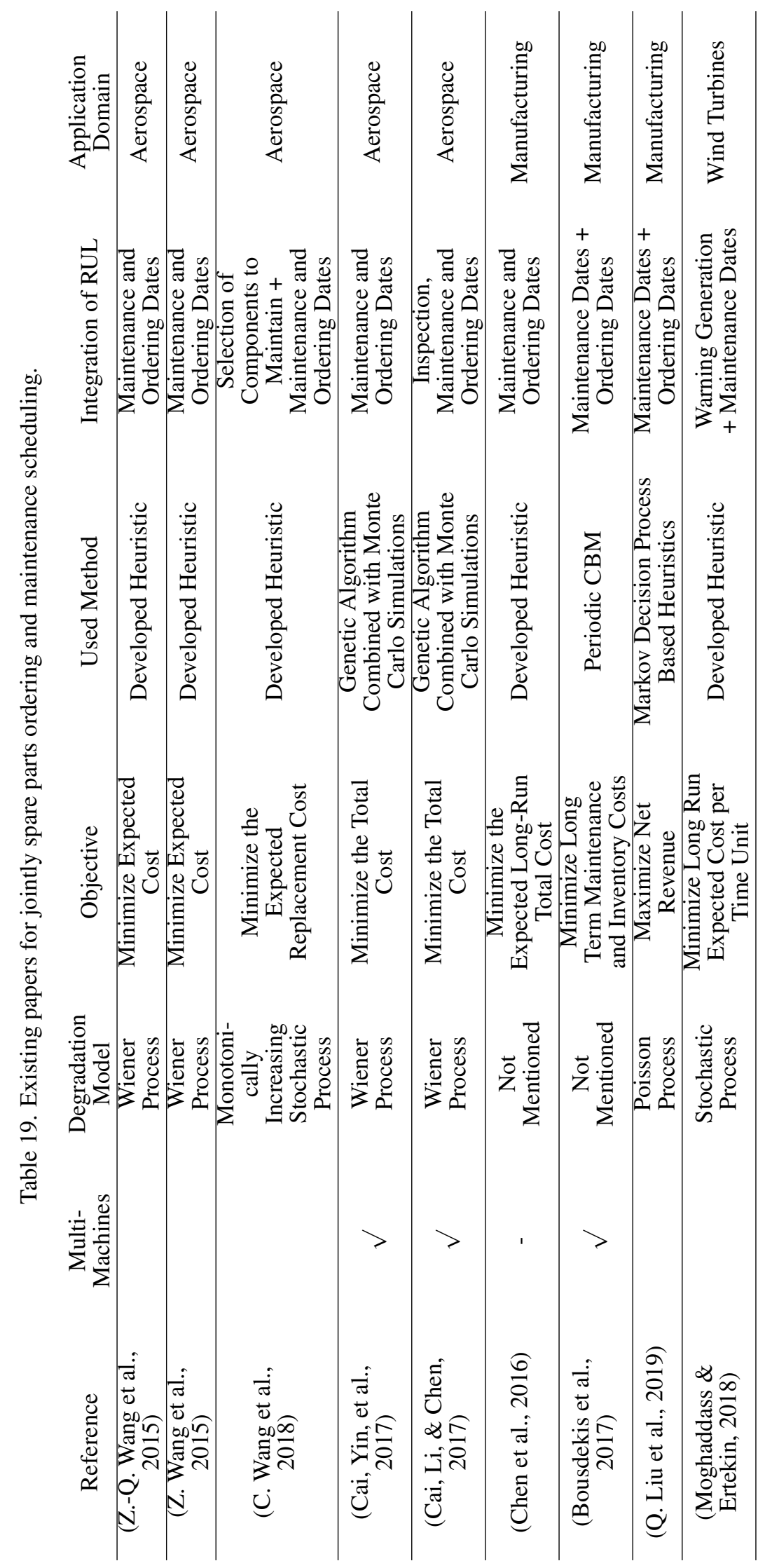




\subsection{Mixed Decisions Framework}

(Choo, Beling, LaViers, Marvel, \& Weiss, 2015) and (Choo, Adams, Weiss, Marvel, \& Beling, 2016) presented a future vision of integrating PHM in the smart manufacturing systems. In this framework, the PHM information is no longer confined to the lowest levels of the control hierarchy, and decisions are no more locally made and limited to a specific component or machine. The proposed adaptive multiscale PHM (AM-PHM) approach for system-wide healthaware decision-making serves as a structure to move health information from components up in the hierarchy until they reach the facility manager and to the emanate control signal in the other way. The AM-PHM also creates operational profiles that include operational policies and projected health information (degradation levels, RUL). The hierarchy goes from component level to facility manager through machines, shops, assembly lines, etc. Each of these nodes is a decisionmaking node influenced by the decisions of the superior level. Later, Choo et al. (Choo, Weiss, \& Beling, 2017) improved the proposed AM-PHM framework by adapting a model-free environment for the nodes. Also, decisions are now passed down from a parent node to their children nodes as an optimization objective through reward weights instead of being transmitted as constraints.

\section{NEW TRENDS AND SCIENTIFIC LOCKS}

Considering the classification of post-prognostics proposed in the previous sections we can now draw a parallel between PHM process and system process. Figure 2 presents the synthesis of the PHM role in the industrial applications. The resource planning bloc is responsible for the definition of the spare parts, raw materials and tool storage and orders. The objectives bloc uses these resources to define the strategy of the system and plan the work to achieve it. Once the tasks are defined, they are transformed into a group of set points used to control the system or the actuator. The closed loop here represents a classic automatic control loop. Aside from the sensors' data inputs, the PHM process acquires the outputs of the system process like the resources status, the objectives of the machines and its control law. These data are considered in either the prognostic process or the decision-making one. Finally, the decisions are used to fine-tune the respective module to the decisions type.

Throughout the analysis of the post-prognostic decisions literature, one can note a lock of positioning between the works. This lack is due to the fact that the papers used different problem formulations (i. e. , objective functions, constraints, etc. . ) plus, they used various metrics to assess the outcomes of the decisions. Thus, it is difficult to position one's work to the current research in the domain if the chosen objective function is not the same. Most authors prefer to compare their results to other maintenance strategies, namely, systematic

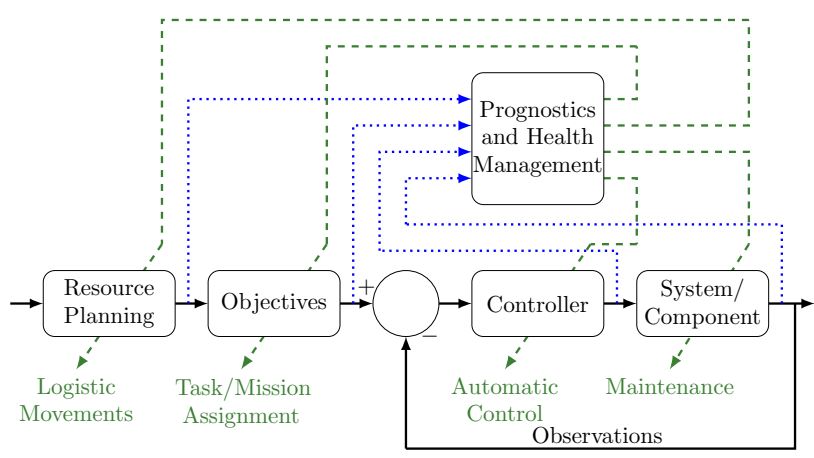

Figure 2. PHM place related to the system process

preventive maintenance, cyclic maintenance and corrective maintenance. Therefore, there is a need to define some common metrics to evaluate decisions and rank research according to these metrics. Ranking the works in the field by different metrics would help identify the gaps in the research and provide support for future work. Thus, the post-prognostic decisions will evolve rapidly.

Another reason behind the lack of positioning in the postprognostic decision-making literature is the lack of information about the decision problem. In most works, the RUL and/or the health indicators of the machine are assumed to be given by a prognostic algorithm and the degradation of the considered components is supposed to be known. However, no further information is given about how to obtain these values or the model used behind to get the predictive information. Since post-prognostic decision-making is based on predictive information, omitting how to get this information and its nature would result in the impossibility to reproduce the same results. This problem could be solved by introducing the method used for the prognostic module and/or the considered degradation model even by just referring to a previous work that studied the prognostic problem. Moreover, in some cases in which the degradation model is given, the parameters of this model are omitted. As an example, in (Camci, 2009), the author gave the degradation model however they did not provide the used numeric parameters in the study. Allowing a transparent share of data in the context of PPDM would facilitate the positioning of work and thus help the evolution of the domain.

The post-prognostic decision-making literature would benefit from the proposition and the use of common metrics to evaluate the outcome of the proposed approaches. Such the case for the prognostic literature, the use of mean squared error and its variants allows to better position the works. However, defining such metrics is hard due to the lack of some information about the decisions in the literature. Moreover, only few works provide full information about their decisionmaking process and performances. Some decision method 
performances are omitted, such as:

- the execution time of the algorithm is an important metric of the used decision method. This execution time would allow readers to check if the proposed method validate the time constraint. It also provide a support for method selection since in some application the reaction time of the decision module is critical.

- the quality of the provided decision define if the provided solution is optimal or not and/or how far is it from the optimal solution. This could be obtained by comparing the proposed methods to exact resolution methods if they could be applied. This metric could be useful in defining the trade off between the quality of the solution and the execution time of the algorithm.

- the effects of RUL uncertainties on decisions and how they are expressed. The prognostic method usually estimate a distribution of possible RUL and/or evolution of the health indicators. However, most works in PPDM assume that the provided predictive information are deterministic. One could question the robustness of these decision methods towards the uncertainties in the prognostic results.

- the type of decision-making process (i. e. , online or offline algorithms); and

- the consequences of the decision on the different work processes, mainly the load of the maintenance shop. Such metric could change the validation process of the proposed solution. For example, in some cases the use of prognostic information in the decision-making process could result in oversizing the maintenance workshop. Hence, at most time the maintenance workload are getting paid but they are not performing any actions. While at some cases, they are overloaded with maintenance interventions. This could be not allowed by the system owner.

The PPDM is expressed as an optimization process of a given problem formulation. However, once the solution is obtained by the optimization algorithm how could one judge if this solution is valid or not? And how can one validate the decisionmaking process? Thus, one important challenge of the postprognostic decision-making process is the validation of the given solution. One possible way of thinking of decision validation process could be by applying the selected decision on the system and assessing its outcome. However, this validation could be expensive and does not allow to compare the applied decision to other possible solutions. One can also think of simulation as a possible validation process. Given the system state and the proposed solution, the outcomes of its application could be simulated. This method allows to compare different decisions. However, building a simulator that models the system could be challenging.

In the decision-making process, the resolution algorithm is building a solution for a given optimization problem. One important parameter of this process is how long far ahead should the solution be? In other words, what is the duration of the decision horizon? On one hand, if the system owner would implement the PPDM for short decision horizon the resolution algorithm would probably consume less time in finding the optimal plan. However, solving the same problem repeatedly over short duration could be seen as locally optimizing a global problem. Plus, the sum of local optimal does not necessary have a global optimal. Besides, the algorithm would base its decision-making process on information in the near future. On the other hand, if large decision horizon is considered, the difference between what the prognostic algorithm estimates and the real values would become greater due to the accumulation of uncertainties over the decision horizon. Besides, the algorithm would take more time to solve the problem. Therefore, a compromise between the resolution method performance and the management of the uncertainty should be studied and defined through the choice of the appropriate decision horizon.

Naturally, one can understand the functioning of the PHM process presented in Figure 3. Thanks to the sensors of a system,data are obtained. The observation of sensor data (storage, signal processing etc. ) allows for producing preprocessed information that can be used in an analysis module (detection, diagnostics and prognostics). The analysis ends with estimated RUL and/or the health indicators (HI). Based on the obtained values, the decision support system provides a schedule of the optimal or sub-optimal decisions. When decisions are applied, they have an effect on the system's states. Changes in the system's states are observed in the sensor data, which cause modification of the prognostics outcomes. With a different RUL and/or HI, a different schedule of decisions is obtained. For each new cycle, a new schedule is obtained.

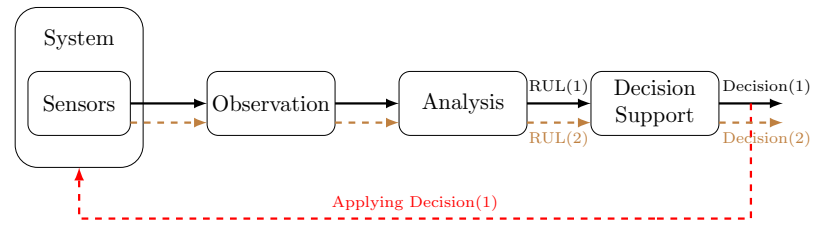

Figure 3. The natural PHM process

Most of the works are based on a single RUL value. In some cases, the decisions are made over a long horizon without considering the effects of the decision on the system. Most of the effects of decisions are defined in assumptions such as the perfect quality of the maintenance or the constant environmental and operational conditions (for example, in (Van Horenbeek \& Pintelon, 2013) or in (Camci, 2009), the production system is supposed to produce the same product and in the same operating conditions). The assumptions 
made about the type of decision limit the approach of PHM to an open loop process. Decisions that are made with such a process can become quickly out of date because their effects are not considered in the health assessment of the systems. Surely the decision-making function is not run just once during the lifetime of the asset. Instead, it is run repeatedly, and the impact of the decisions is captured through this cyclic execution. Yet, this point was not mentioned in most of the literature. Moreover, the suitable duration for the decision horizon or the frequency of the decision-making was not studied. Consequently, we can suppose that these papers considers the open loop PHM process as presented in Figure 4. In Figure 4, the decision applying process is represented by a dashed gray line since it is a natural process but was not explicitly mentioned or studied.

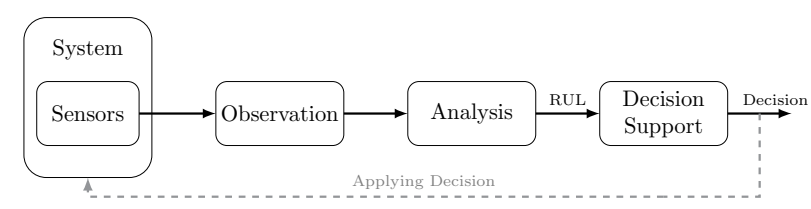

Figure 4. The proposed PHM process in most literature works

Although the importance of integrating decisions in the prognostics module was not the aim of previous studies, some works have included the hypothetical decisions or future loads into the prognostics procedure. Both Daigle and Goebel (Daigle \& Goebel, 2010) and Zhang et al. (Zhang et al., 2014) integrated information about the future loads of the system in their model-based prognostic methods to estimate the RUL of the system. In (Zhang et al., 2014), the resulting RUL was more involved in the decision-making process by using it as a variable in the optimization objective function. Welz et al. (Welz, Coble, Upadhyaya, \& Hines, 2017) integrated maintenance information into the prognostic process. Maintenance actions, whether perfect or imperfect, were modeled and then used as inputs to the prognostics. The approach was tested for the Weibull method and the general path model (GPM) method. In both, the integration of the maintenance model improved the accuracy of the prediction by reducing the prediction error. The approach was validated on a heat exchanger test bed. Vileiniskis and RemenytePrescott (Vileiniskis \& Remenyte-Prescott, 2017) developed a new approach for predicting the quantitative risk of failure. The method consists of creating a Petri-net model to present the current state of the system, the degradation and the future operation and maintenance activities. The model is then run in a Monte Carlo simulation to obtain the statistics of components' performance over a selected horizon. The results are fed into a bow-tie model to estimate the risk of hazardous events.

By analyzing the papers that treated post-prognostic decision- making and those that aimed to integrate future loads in the RUL prediction, we can easily notice the important interactions between the prognostics and the decision-making modules. In Figure 5, the prognostics module influences the decision by the value of the RUL, and the decision-making process modifies the prognostics outcome with the future loads and the selected decisions. This can drive toward the necessity of integrating the prognostics and decision processes into a closed loop. One proven advantage of such an approach is the improved accuracy of the RUL prediction.

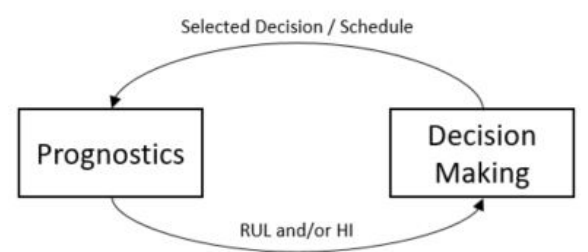

Figure 5. The interactions between the prognostics and the decision-making modules

\section{Conclusion}

In this article, the literature related to prognostic-based decision-making is reviewed. The related works were classified according to the decision type into three categories: (a) maintenance decisions, (b) operational decisions, for which we proposed three sub-categories: (i) automatic control; (ii) task/mission assignment; and (iii) logistic decisions; and (c) mixed decisions that include maintenance and operational decisions jointly optimized. The main assumptions and applications were synthesized from the articles for each category. Finally, we presented the new trends in the PHM field by emphasizing the relationship and interactions between the prognostics and the decision-making processes.

The importance of those interactions offers a promising field of research in prognostics and health management. For this reason, we will aim our future works at defining new approaches to a closed loop PHM process to emphasize the prognostics-decision-making loop.

\section{REFERENCES}

Aizpurua, J., Catterson, V., Papadopoulos, Y., Chiacchio, F., \& D'Urso, D. (2017, December). Supporting group maintenance through prognostics-enhanced dynamic dependability prediction. Reliability Engineering and System Safety, 168, 171-188.

Ambani, S., Li, L., \& Ni, J. (2009). Condition-Based Maintenance Decision-Making for Multiple Machine Systems. Journal of Manufacturing Science and Engineering, 131(3), 031009.

Balaban, E., \& Alonso, J. J. (2012). An approach to prognos- 
tic decision making in the aerospace domain. In Annual conference of the prognostics and health management society.

Benaggoune, K., Meraghni, S., Ma, J., Mouss, L., \& Zerhouni, N. (2020). Post prognostic decision for predictive maintenance planning with remaining useful life uncertainty. In 2020 prognostics and health management conference (phm-besançon) (pp. 194-199).

Bencheikh, G., Letouzey, A., \& Desforges, X. (2018, august). Process for joint scheduling based on health assessment of technical resources. In 10th ifac symposium on fault detection, supervision and safety for technical processes safeprocess 2018 (Vol. 51, pp. 192-199). Warsaw, Poland.

Bogdanov, A., Chiu, S., Gokdere, L. U., \& Vian, J. (2007, May). Stochastic Optimal Control of a Servo Motor with a Lifetime Constraint. In Proceedings of the 45th IEEE Conference on Decision and Control. San Diego, CA, USA: IEEE.

Bole, B., Tang, L., Goebel, K., \& Vachtsevanos, G. (2011, July). Adaptive Load-Allocation for Prognosis-Based Risk Management. In Annual Conference of the Prognostics and Health Management Society, 2011. Montreal, Canada.

Bougacha, O., Varnier, C., Zerhouni, N., \& Hajri-Gabouj, S. (2018, dec). A post-prognostic decision approach for production and maintenance planning. In 48th conference on computers \& industrial engineering (2018).

Bousdekis, A., Papageorgiou, N., Magoutas, B., Apostolou, D., \& Mentzas, G. (2017). A Proactive Event-driven Decision Model for Joint Equipment Predictive Maintenance and Spare Parts Inventory Optimization. Procedia CIRP, 59, 184-189.

Brown, D. W., Georgoulas, G., Bole, B., Pei, H.-L., Orchard, M., Tang, L., ... Vachtsevanos, G. (2009, September). Prognostics Enhanced Reconfigurable Control of Electro-Mechanical Actuators. In Annual Conference of the Prognostics and Health Management Society, 2009. San Diego, CA, USA.

Brown, D. W., \& Vachtsevanos, G. (n.d., July). A Prognostic Health Management Based Framework for FaultTolerant Control. In Annual Conference of the Prognostics and Health Management Society, 2011. Montreal, Canada.

Byington, C. S., Roemer, M. J., \& Galie, T. (2002). Prognostic enhancements to diagnostic systems for improved condition-based maintenance [military aircraft]. In Proceedings, ieee aerospace conference, 2002. IEEE.

Cai, J., Li, X., \& Chen, X. (2016). Optimization of Aeroengine Shop Visit Decisions Based on Remaining Useful Life and Stochastic Repair Time. Mathematical Problems in Engineering, 2016, 1-11.

Cai, J., Li, X., \& Chen, X. (2017, December). Joint optimization of maintenance inspection and spare provisioning for aircraft deteriorating parts. Journal of Systems Engineering and Electronics, 28(6), 1133 - 1140.

Cai, J., Yin, Y., Zhang, L., \& Chen, X. (2017). Joint Optimization of Preventive Maintenance and Spare Parts Inventory with Appointment Policy. Mathematical Problems in Engineering, 2017, 1-12.

Camci, F. (2009, September). System Maintenance Scheduling With Prognostics Information Using Genetic Algorithm. IEEE Transactions on Reliability, 58(3), 539552.

Camci, F. (2014, September). The travelling maintainer problem: integration of condition-based maintenance with the travelling salesman problem. Journal of the Operational Research Society, 65(9), 1423-1436.

Camci, F. (2015, September). Maintenance scheduling of geographically distributed assets with prognostics information. European Journal of Operational Research, 245(2), 506-516.

Chebel-Morello, B., Nicod, J.-M., \& Varnier, C. (2017). From Prognostics and Health Systems Management to Predictive Maintenance 2. John Wiley \& Sons.

Chen, X., Xu, D., \& Xiao, L. (2016, December). Joint optimization of replacement and spare ordering for critical rotary component based on condition signal to date. Eksploatacja i Niezawodnosc - Maintenance and Reliability, 19(1), 76-85.

Cheng, G. Q., Zhou, B. H., \& Li, L. (2018, July). Integrated production, quality control and condition-based maintenance for imperfect production systems. Reliability Engineering and System Safety, 175, 251-264.

Cholette, M. E., Celen, M., Djurdjanovic, D., \& Rasberry, J. D. (2013, November). Condition Monitoring and Operational Decision Making in Semiconductor Manufacturing. IEEE Transactions on Semiconductor Manufacturing, 26(4), 454-464.

Choo, B. Y., Adams, S. C., Weiss, B. A., Marvel, J. A., \& Beling, P. A. (2016). Adaptive Multi-scale Prognostics and Health Management for Smart Manufacturing Systems. International Journal of Prognostics and Health Management.

Choo, B. Y., Beling, P. A., LaViers, A. E., Marvel, J. A., \& Weiss, B. A. (2015). Adaptive Multi-scale PHM for Robotic Assembly Processes. In Proceedings of the Annual Conference in Prognostics and Health Management Society. San Diego, CA, USA.

Choo, B. Y., Weiss, B. A., \& Beling, P. A. (2017, June). Health-Aware Hierarchical Control for Smart Manufacturing using Reinforcement Learning. In $\{$ Proceedings of the IEEE International Conference on Prognostics and Health Management. Dallas, TX, USA: IEEE.

Chrétien, S., Herr, N., Nicod, J.-M., \& Varnier, C. (2016, october). Post-Prognostics Decision for Optimizing the Commitment of Fuel Cell Systems. In 3rd ifac work- 
shop on advanced maintenance engineering, services and technology amest 2016 (Vol. 49, pp. 168-173). Biarritz, France.

Cui, Y., Shi, J., \& Wang, Z. (2015, September). Discrete Event Logistics Systems (DELS) simulation modeling incorporating two-step Remaining Useful Life (RUL) estimation. Computers in Industry, 72, 68-81.

Daigle, M., \& Goebel, K. (2010). improving computational efficiency of prediction in model-based prognostics using the unscented transform. Annual Conference of the Prognostics and Health Management Society 2010.

De Medeiros, I. P., Rodrigues, L. R., Kern, C. S., Dos Santos, R. D. C., \& Shiguemori, E. H. (2015). Integrated task assignment and maintenance recommendation based on system architecture and PHM information for UAVs. In Systems Conference (SysCon), 2015 9th Annual IEEE International (pp. 182-188). IEEE.

de Medeiros, I. P., Rodrigues, L. R., Santos, R., Shiguemori, E. H., \& Júnior, C. L. N. (2014). PHM-based Multi-UAV task assignment. In Systems Conference (SysCon), 2014 8th Annual IEEE (pp. 42-49). IEEE.

Desforges, X., Diévart, M., \& Archimède, B. (2017, April). A prognostic function for complex systems to support production and maintenance co-operative planning based on an extension of object oriented Bayesian networks. Computers in Industry, 86, 34-51.

Do, P., Voisin, A., Levrat, E., \& Iung, B. (2015, January). A proactive condition-based maintenance strategy with both perfect and imperfect maintenance actions. Reliability Engineering and System Safety, 133, 22-32.

Durazo-Cardenas, I., Starr, A., Turner, C. J., Tiwari, A., Kirkwood, L., Bevilacqua, M., ... Emmanouilidis, C. (2018, April). An autonomous system for maintenance scheduling data-rich complex infrastructure: Fusing the railways' condition, planning and cost. Transportation Research Part C: Emerging Technologies, 89, 234-253.

Feng, D., Lin, S., He, Z., \& Sun, X. (2017, July). A technical framework of $\mathrm{phm}$ and active maintenance for modern high-speed railway traction power supply systems. International Journal of Rail Transportation, 5(3), 145169.

Feng, Q., Bi, X., Zhao, X., Chen, Y., \& Sun, B. (2017, January). Heuristic hybrid game approach for fleet condition-based maintenance planning. Reliability Engineering and System Safety, 157, 166-176.

Fitouri, C., Fnaiech, N., Varnier, C., Fnaiech, F., \& Zerhouni, N. (2016). A Decison-Making Approach for Job Shop Scheduling with Job Depending Degradation and PredictiveMaintenance. In (Vol. 49, pp. 1490-1495). Troyes, France.

Goebel, K., Celaya, J., Sankararaman, S., Roychoudhury, I., Daigle, M. J., \& Saxena, A. (2017). Prognostics: The Science of Making Predictions. CreateSpace Indepen- dent Publishing Platform.

Gouriveau, R., Medjaher, K., \& Zerhouni, N. (2016). From Prognostics and Health Systems Management to Predictive Maintenance 1. John Wiley \& Sons.

Griffith, D. T., Yoder, N. C., Resor, B., White, J., \& Paquette, J. (2014, November). Structural health and prognostics management for the enhancement of offshore wind turbine operations and maintenance strategies: Structural health and prognostics management for offshore O\&M. Wind Energy, 17(11), 1737-1751.

Grosso, J. M., Ocampo-Martinez, C., \& Puig, V. (2016, January). Reliability-based economic model predictive control for generalised flow-based networks including actuators' health-aware capabilities. International Journal of Applied Mathematics and Computer Science, 26(3). doi: 10.1515/amcs-2016-0044

Haddad, G., Peter, S., \& Pecht, M. (2011). A Real Options Optimization Model to Meet Availability Requirements for Offshore Wind Turbines. In Proceedings of MFPT: The Applied Systems Health Management Conference. Virginia Beach, VA, USA.

Herr, N. (2015). Post-Prognostic scheduling of heterogeneous distributed platforms (Unpublished doctoral dissertation). Franche-Comte University, Besancon, France.

Herr, N., Nicod, J.-M., \& Varnier, C. (2014). Prognosticsbased scheduling in a distributed platform: Model, complexity and resolution. In Automation Science and Engineering (CASE), 2014 IEEE International Conference on (pp. 1054-1059). IEEE.

Herr, N., Nicod, J.-M., Varnier, C., Jardin, L., Sorrentino, A., Hissel, D., \& Péra, M.-C. (2017, May). Decision process to manage useful life of multi-stacks fuel cell systems under service constraint. Renewable Energy, 105, 590-600.

Herr, N., Nicod, J.-M., Varnier, C., Zerhouni, N., Cherif, M., \& Fnaiech, N. (2017). Joint optimization of train assignment and predictive maintenance scheduling. Lille, France.

Huynh, K., Grall, A., \& Bérenguer, C. (2017, March). Assessment of diagnostic and prognostic condition indices for efficient and robust maintenance decisionmaking of systems subject to stress corrosion cracking. Reliability Engineering and System Safety, 159, $237-$ 254.

Huynh, K. T., Barros, A., \& Berenguer, C. (2015, March). Multi-Level Decision-Making for The Predictive Maintenance of \$ $\$$-Out-of-\$n \$ :F Deteriorating Systems. IEEE Transactions on Reliability, 64(1), 94117.

Huynh, K. T., Castro, I. T., Barros, A., \& Berenguer, C. (2014, July). On the Use of Mean Residual Life as a Condition Index for Condition-Based Maintenance Decision-Making. IEEE Transactions on Systems, Man, and Cybernetics: Systems, 44(7), 877-893. 
Iyer, N., Goebel, K., \& Bonissone, P. (2006). Framework for post-prognostic decision support. In Aerospace Conference, 2006 IEEE (pp. 10-pp). IEEE.

Jain, A. K., \& Lad, B. K. (2017, June). Dynamic Optimization of Process Quality Control and Maintenance Planning. IEEE Transactions on Reliability, 66(2), 502517.

Jin, C., Djurdjanovic, D., Ardakani, H. D., Wang, K., Buzza, M., Begheri, B., ... Lee, J. (2015). A comprehensive framework of factory-to-factory dynamic fleetlevel prognostics and operation management for geographically distributed assets. In Automation Science and Engineering (CASE), 2015 IEEE International Conference on (pp. 225-230). IEEE.

Julka, N., Thirunavukkarasu, A., Lendermann, P., Gan, B. P., Schirrmann, A., Fromm, H., \& Wong, E. (2011, August). Making use of prognostics health management information for aerospace spare components logistics network optimisation. Computers in Industry, 62(6), 613-622.

Khoury, E., Deloux, E., Grall, A., \& Bérenguer, C. (2013). On the Use of Time-Limited Information for Maintenance Decision Support: A Predictive Approach under Maintenance Constraints. Mathematical Problems in Engineering, 2013, 1-11.

Ladj, A., Benbouzid-Si Tayeb, F., Varnier, C., Dridi, A. A., \& Selmane, N. (2017). A Hybrid of Variable Neighbor Search and Fuzzy Logic for the permutation flowshop scheduling problem with predictive maintenance. Procedia Computer Science, 112, 663-672.

Ladj, A., Varnier, C., Tayeb, F. B. S., \& Zerhouni, N. (2017). Exact and heuristic algorithms for post prognostic decision in a single multifunctional machine. International Journal of of Prognostics and Health Management, 8(2).

Langeron, Y., Fouladirad, M., \& Grall, A. (2016, june). Controlled systems, failure prediction and maintenance. In 8th ifac conference on manufacturing modelling, management and control mim (Vol. 49, pp. 805-808). Troyes, France.

Langeron, Y., Grall, A., \& Barros, A. (2013). Actuator Health Prognosis for Designing LQR Control in Feedback Systems. Chemical engineering transactions, 33. doi: 10.3303/CET1333164

Langeron, Y., Grall, A., \& Barros, A. (2015, August). A modeling framework for deteriorating control system and predictive maintenance of actuators. Reliability Engineering and System Safety, 140, 22-36.

Langeron, Y., Grall, A., \& Barros, A. (2017, August). Joint maintenance and controller reconfiguration policy for a gradually deteriorating control system. Proceedings of the Institution of Mechanical Engineers, Part O: Journal of Risk and Reliability, 231(4), 339-349.

Lebold, M., \& Thruston, M. (2001, January). Open Stan- dards For Condition-Based Maintenance and Prognostic Systems. In Maintenance and Reliability Conference (MARCON). Gatlinburg, USA.

Lei, X., \& Sandborn, P. A. (2016). PHM-based wind turbine maintenance optimization using real options. International Journal of of Prognostics and Health Management, 7(1), 1-14.

Lei, X., \& Sandborn, P. A. (2018, February). Maintenance scheduling based on remaining useful life predictions for wind farms managed using power purchase agreements. Renewable Energy, 116, 188-198.

Li, R., \& Ryan, J. K. (2011, September). A Bayesian Inventory Model Using Real-Time Condition Monitoring Information. Production and Operations Management, 20(5), 754-771.

Li, Z., Guo, J., \& Zhou, R. (2016). Maintenance scheduling optimization based on reliability and prognostics information. In Reliability and Maintainability Symposium (RAMS), 2016 Annual (pp. 1-5). IEEE.

Lin, L., Luo, B., \& Zhong, S. (2017, December). Development and application of maintenance decision-making support system for aircraft fleet. Advances in Engineering Software, 114, 192-207.

Lin, S., Zhang, A., \& Feng, D. (2016, October). Maintenance decision-making model based on POMDP for traction power supply equipment and its application. In 2016 Prognostics and System Health Management Conference (PHM-Chengdu) (pp. 1-6). Chengdu, China: IEEE.

Lin, X., Basten, R., Kranenburg, A., \& van Houtum, G. (2017, December). Condition based spare parts supply. Reliability Engineering and System Safety, 168, 240-248.

Liu, Q., Dong, M., Chen, F., Lv, W., \& Ye, C. (2019, February). Single-machine-based joint optimization of predictive maintenance planning and production scheduling. Robotics and Computer-Integrated Manufacturing, 55, 173-182.

Liu, Q., Dong, M., Lv, W., \& Ye, C. (2017, February). Manufacturing system maintenance based on dynamic programming model with prognostics information. Journal of Intelligent Manufacturing.

Liu, X., Yang, T., Pei, J., Liao, H., \& Pohl, E. A. (2019, March). Replacement and Inventory Control for a Multi-Customer Product Service System with Decreasing Replacement Costs. European Journal of Operational Research, 273(2), 561-574.

Luo, B., \& Lin, L. (2018). Multi-objective decision-making model based on CBM for an aircraft fleet. Xi'an City, China.

Matyas, K., Nemeth, T., Kovacs, K., \& Glawar, R. (2017). A procedural approach for realizing prescriptive maintenance planning in manufacturing industries. CIRP Annals, 66(1), 461-464. 
Mazidi, P., Bertling Tjernberg, L., \& Sanz Bobi, M. A. (2017, April). Wind turbine prognostics and maintenance management based on a hybrid approach of neural networks and a proportional hazards model. Proceedings of the Institution of Mechanical Engineers, Part O: Journal of Risk and Reliability, 231(2), 121-129.

Meraghni, S., Terrissa, L. S., Ayad, S., Zerhouni, N., \& Varnier, C. (2018, March). Post-prognostics decision in cyber-physical systems. In 2018 International Conference on Advanced Systems and Electric Technologies (IC_aset) (pp. 201-205). Hammamet: IEEE.

Meraghni, S., Terrissa, L. S., Zerhouni, N., Varnier, C., \& Ayad, S. (2016). A post-prognostics decision framework for cell site using Cloud computing and Internet of Things. In Cloud Computing Technologies and Applications (CloudTech), 2016 2nd International Conference on (pp. 310-315). IEEE.

Moghaddass, R., \& Ertekin, S. (2018, April). Joint optimization of ordering and maintenance with condition monitoring data. Annals of Operations Reseach, 263(1), 271-310.

Nguyen, D. N., Dieulle, L., \& Grall, A. (2014, August). Feedback Control System with Stochastically Deteriorating Actuator: Remaining Useful Life Assessment. In Proceedings of the 19th World Congress The International Federation of Automatic Control. Cape Town, South Africa.

Nguyen, K.-A., Do, P., \& Grall, A. (2015, December). Multilevel predictive maintenance for multi-component systems. Reliability Engineering and System Safety, 144, 83-94.

Nguyen, K. T., \& Medjaher, K. (2019). A new dynamic predictive maintenance framework using deep learning for failure prognostics. Reliability Engineering \& System Safety, 188, 251-262.

Niknam, S. A., Kobza, J. E., \& Hines, J. W. (2015). Operation and maintenance decision-making using prognostic information. In Reliability and Maintainability Symposium (RAMS), 2015 Annual (pp. 1-7). IEEE.

Niu, G., \& Jiang, J. (2017). Prognostic control-enhanced maintenance optimization for multi-component systems. Reliability Engineering \& System Safety, 168, 218-226.

Niu, G., \& Liu, S. (2018). Demagnetization monitoring and life extending control for permanent magnet-driven traction systems. Mechanical Systems and Signal Processing, 103, 264-279.

Nzukam, C., Voisin, A., Levrat, E., Sauter, D., \& Iung, B. (2017, July). A dynamic maintenance decision approach based on maintenance action grouping for HVAC maintenance costs savings in Non-residential buildings. In 20th ifac world congress (Vol. 50, pp. 13722-13727).

Nzukam, C., Voisin, A., Levrat, E., Sauter, D., \& Iung, B.
(2018, june). Opportunistic maintenance scheduling with stochastic opportunities duration in a predictive maintenance strategy. In 16th ifac symposium on information control problems in manufacturing incom (Vol. 51, pp. 453-458). Bergamo, Italy.

Pan, E., Liao, W., \& Xi, L. (2012, June). A joint model of production scheduling and predictive maintenance for minimizing job tardiness. The International Journal of Advanced Manufacturing Technology, 60(9), 10491061.

Pereira, E. B., Galvão, R. K. H., \& Yoneyama, T. (2010). Model predictive control using prognosis and health monitoring of actuators. In Industrial Electronics (ISIE), 2010 IEEE International Symposium on (pp. 237-243). IEEE.

Ramos Rodrigues, L., Paixao de Medeiros, I., \& Strottmann Kern, C. (2015, April). Maintenance cost optimization for multiple components using a condition based method. In 2015 Annual IEEE Systems Conference (SysCon) Proceedings (pp. 164-169). Vancouver, BC, Canada: IEEE.

Rodrigues, L. R., Gomes, J. P. P., \& Alcântara, J. F. L. (2018, May). Embedding Remaining Useful Life Predictions into a Modified Receding Horizon Task Assignment Algorithm to Solve Task Allocation Problems. Journal of Intelligent \& Robotic Systems, 90(1-2), 133-145.

Rodrigues, L. R., Gomes, J. P. P., Ferri, F. A. S., Medeiros, I. P., Galvao, R. K. H., \& Nascimento Junior, C. L. (2015, December). Use of PHM Information and System Architecture for Optimized Aircraft Maintenance Planning. IEEE Systems Journal, 9(4), 1197-1207.

Shi, H., \& Zeng, J. (2016, March). Real-time prediction of remaining useful life and preventive opportunistic maintenance strategy for multi-component systems considering stochastic dependence. Computers \& Industrial Engineering, 93, 192-204.

Si, X., Li, T., Zhang, Q., \& Hu, X. (2018, September). An Optimal Condition-Based Replacement Method for Systems With Observed Degradation Signals. IEEE Transactions on Reliability, 67(3), 1281-1293.

Skima, H. (2016). Prognostics and distributed algorithms for post-prognostic decision making in MEMS based Systems (Unpublished doctoral dissertation). FrancheComte University, Besancon, France.

Skima, H., Varnier, C., Dedu, E., Medjaher, K., \& Bourgeois, J. (2017, February). Post-prognostics decision making in distributed MEMS-based systems. Journal of Intelligent Manufacturing.

Sun, B., Zeng, S., Kang, R., \& Pecht, M. (2010, January). Benefits analysis of prognostics in systems. 2010 Prognostics and System Health Management Conference, $1-10$.

Tamssaouet, F., Nguyen, K., \& Medjaher, K. (2019). System remaining useful life maximization through mis- 
sion profile optimization. In Asia-pacific conference of the prognostics and health management society.

Tang, D., Makis, V., Jafari, L., \& Yu, J. (2015, February). Optimal maintenance policy and residual life estimation for a slowly degrading system subject to condition monitoring. Reliability Engineering and System Safety, 134, 198-207.

Tang, L., Hettler, E., Zhang, B., \& DeCastro, J. (2011, July). A Testbed for Real-Time Autonomous Vehicle PHM and Contingency Management Applications. In Annual Conference of the Prognostics and Health Management Society, 2011. Montreal, Canada.

Tian, Z., Jin, T., Wu, B., \& Ding, F. (2011, May). Condition based maintenance optimization for wind power generation systems under continuous monitoring. Renewable Energy, 36(5), 1502-1509.

Tian, Z., \& Liao, H. (2011, May). Condition based maintenance optimization for multi-component systems using proportional hazards model. Reliability Engineering and System Safety, 96(5), 581-589.

Uckun, S., Goebel, K., \& Lucas, P. J. (2008, October). Standardizing research methods for prognostics. In International Conference on Prognostics and Health Management, 2008. PHM 2008., 1-10.

Vachtsevanos, G., \& Wang, P. (2001, August). Fault prognosis using dynamic wavelet neural networks. In IEEE Autotestcon Proceedings. IEEE Systems Readiness Technology Conference. (pp. 857-870). Valley Forge, PA, USA.

Van Horenbeek, A., \& Pintelon, L. (2013, December). A dynamic predictive maintenance policy for complex multi-component systems. Reliability Engineering and System Safety, 120, 39-50.

Verbert, K., De Schutter, B., \& Babuška, R. (2017, March). Timely condition-based maintenance planning for multi-component systems. Reliability Engineering and System Safety, 159, 310-321.

Vianna, W. O. L., \& Yoneyama, T. (2018, June). Predictive Maintenance Optimization for Aircraft Redundant Systems Subjected to Multiple Wear Profiles. IEEE Systems Journal, 12(2), 1170-1181.

Vieira, J. P., Galvão, R. K. H., \& Yoneyama, T. (2015, December). Predictive Control for Systems with Loss of Actuator Effectiveness Resulting from Degradation Effects. Journal of Control, Automation and Electrical Systems, 26(6), 589-598.

Vileiniskis, M., \& Remenyte-Prescott, R. (2017). Quantitative risk prognostics framework based on petri net and bow-tie models. Reliability Engineering and System Safety, 165, 62-73.

Villarejo, R., Johansson, C.-A., Galar, D., Sandborn, P., \& Kumar, U. (2016, July). Context-driven decisions for railway maintenance. Proceedings of the Institution of Mechanical Engineers, Part F: Journal of Rail and
Rapid Transit, 230(5), 1469-1483.

Wang, C., Xu, J., Wang, H., \& Zhang, Z. (2018, September). A criticality importance-based spare ordering policy for multi-component degraded systems. Eksploatacja i Niezawodnosc - Maintenance and Reliability, 20(4), 662-670.

Wang, K., Tian, J., Pecht, M., \& Xu, A. (2015). A Prognostics and Health Management Based Method for Refurbishment Decision Making for Electromechanical Systems. In 15th ifac symposium oninformation control problems inmanufacturing (Vol. 48, pp. 454-459).

Wang, P., Tamilselvan, P., Twomey, J., \& Youn, B. D. (2013, June). Prognosis-informed wind farm operation and maintenance for concurrent economic and environmental benefits. International Journal of Precision Engineering and Manufacturing, 14(6), 1049-1056.

Wang, W. (2014, October). A scheduling model for systems with task and health dependent remaining useful life prognostics. International Journal of Production Research, 52(19), 5764-5779. doi: 10.1080/00207543.2014.910629

Wang, Y., Gogu, C., Binaud, N., Bes, C., Haftka, R. T., \& Kim, N. H. (2017, June). A cost driven predictive maintenance policy for structural airframe maintenance. Chinese Journal of Aeronautics, 30(3), 12421257.

Wang, Y., Gogu, C., Binaud, N., Bes, C., Haftka, R. T., \& Kim, N.-H. (2018, December). Predictive airframe maintenance strategies using model-based prognostics. Proceedings of the Institution of Mechanical Engineers, Part O: Journal of Risk and Reliability, 232(6), 690-709.

Wang, Z., Hu, C., Wang, W., Kong, X., \& Zhang, W. (2015, August). A prognostics-based spare part ordering and system replacement policy for a deteriorating system subjected to a random lead time. International Journal of Production Research, 53(15), 4511-4527.

Wang, Z.-Q., Wang, W., Hu, C.-H., Si, X.-S., \& Zhang, W. (2015, June). A Prognostic-Information-Based Order-Replacement Policy for a Non-Repairable Critical System in Service. IEEE Transactions on Reliability, 64(2), 721-735.

Welz, Z., Coble, J., Upadhyaya, B., \& Hines, W. (2017, August). Maintenance-based prognostics of nuclear plant equipment for long-term operation. Nuclear Engineering and Technology, 49(5), 914-919.

Yang, Z. M., Djurdjanovic, D., \& Ni, J. (2008, February). Maintenance scheduling in manufacturing systems based on predicted machine degradation. Journal of Intelligent Manufacturing, 19(1), 87-98.

Zhang, B., Tang, L., Decastro, J., \& Goebel, K. (2011). Prognostics-enhanced receding horizon mission planning for field unmanned vehicles. In Aiaa guidance, navigation, and control conference (p. 6294). 
Zhang, B., Tang, L., DeCastro, J., Roemer, M., \& Goebel, K. (2014). Autonomous vehicle battery state-of-charge prognostics enhanced mission planning. International Journal of of Prognostics and Health Management, 5, $1-11$.

Zhao, X., Fouladirad, M., Bérenguer, C., \& Bordes, L. (2009). Maintenance policy for deteriorating system with explanatory variables. R\&RATA.

Zuo, J., Cadet, C., Li, Z., Bérenguer, C., \& Outbib, R. (2020). Post-prognostics decision making for a two-stacks fuel cell system based on a load-dependent deterioration model. In European conference of the prognostics and health management (phm) society (Vol. 5, p. 9).

\section{BIOGRAPHIES}

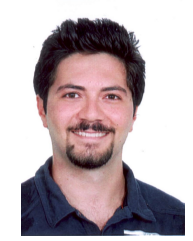

Omar Bougacha Dr. Omar Bougacha obtained his $\mathrm{PhD}$ degree in computer science at the University of Bourgogne FrancheComté. He obtained his engineering degree in 2017 from the National Institute of Applied Science and Technologies in Tunis Tunisia. His $\mathrm{PhD}$ is mainly about decisionmaking in the context of Prognostic and Health Management.

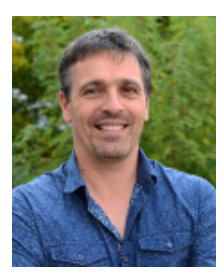

Christophe Varnier Dr. Christophe Varnier is an Associate Professor at ENSMM, France. He obtained his $\mathrm{PhD}$ degree in 1996 at the University of Franche-Comte in Besançon. He is teaching computer science at ENSMM since 1996. $\mathrm{He}$ is a Researcher at the Automatic Control and Micro- Mechatronic Systems Department of the FEMTO-ST Institute. His research interests include Prognostic and Health Management, operation research, scheduling and optimization.

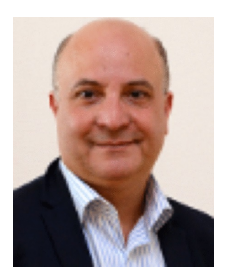

Noureddine Zerhouni Professor Noureddine Zerhouni received his engineer degree from National Engineers and Technicians School of Algiers (ENITA) in 1985. He received his Ph. D. Degree in Automatic Control from the Grenoble National Polytechnic Institute in 1991. In September 1991, he joined the National Engineering School of Belfort (ENIB) as Associate Professor. Since September 1999, Noureddine Zerhouni is Professor at the national high school of mechanics and microtechniques of Besançon. His main research activities are concerned with intelligent maintenance systems and e-maintenance. 